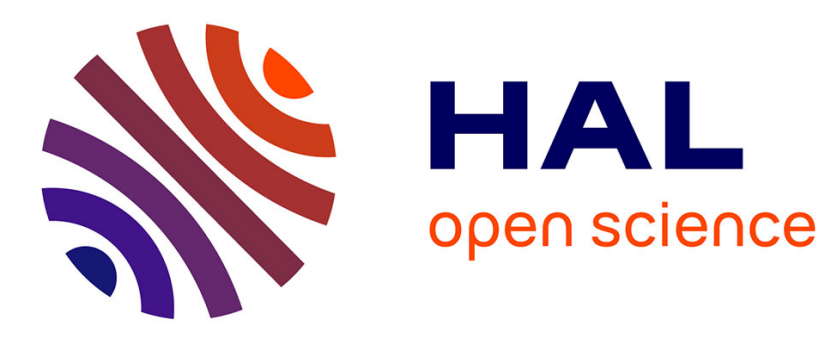

\title{
Des cyclones et des hommes
}

Fabrice Chauvin, Jean-Francois Royer

\section{To cite this version:}

Fabrice Chauvin, Jean-Francois Royer. Des cyclones et des hommes. La Météorologie, 2008, 8e série (61), pp.PP.52-66. meteo-00355587

\section{HAL Id: meteo-00355587 https://hal-meteofrance.archives-ouvertes.fr/meteo-00355587}

Submitted on 7 Nov 2013

HAL is a multi-disciplinary open access archive for the deposit and dissemination of scientific research documents, whether they are published or not. The documents may come from teaching and research institutions in France or abroad, or from public or private research centers.
L'archive ouverte pluridisciplinaire HAL, est destinée au dépôt et à la diffusion de documents scientifiques de niveau recherche, publiés ou non, émanant des établissements d'enseignement et de recherche français ou étrangers, des laboratoires publics ou privés. 


\section{Résumé}

Le nombre global annuel de phénomènes cycloniques est à peu près stable et se situe aux alentours de quatre-vingts systèmes. Mais leur nombre dans chacun des bassins océaniques varie en fonction de la variabilité climatique naturelle, en particulier en fonction du phénomène Enso (El Nino Southern Oscillation). Depuis la saison cyclonique 2005, qui a été intense dans le bassin Atlantique, la question de savoir dans quelle mesure l'activité cyclonique risque d'être modulée par les bouleversements engendrés par le réchauffement anthropique en cours se pose de manière accrue. Le manque de finesse des simulations climatiques globales actuelles ne permet généralement pas de rendre compte du comportement des ces phénomènes cycloniques et, pour l'instant, les études scientifiques n'ont pas encore convergé. Le $4^{\mathrm{e}}$ rapport du Giec fait état du manque de consensus au sein de la communauté scientifique sur cette question. De plus, les bases de données observées sur les systèmes cycloniques ne sont pas assez homogènes pour permettre de détecter un éventuel changement de leur activité dans le passé. Il faudra donc attendre encore quelques années avant de pouvoir se prononcer plus clairement sur ce sujet.

\section{Abstract}

\section{Tropical cyclones and mankind}

The annual global number of tropical cyclones is roughly stable at about $\mathbf{8 0}$. But their number in each of the oceanic basins fluctuates with natural climate variability and particularly with ENSO (El Niño Southern Oscillation). Since the severe 2005 Atlantic cyclonic season, the question of knowing how much impact the current anthropogenic warming will have on cyclonic activity has arisen. The coarse resolution of global climatic simulations does not generally allow to describe their behavior and scientific studies have not yet converged. The 4th IPCC assessment report emphasizes the lack of consensus in the scientific community on this matter. Moreover, databases of observed tropical cyclones are not homogeneous enough to allow detection of any changes in the past. Many more years of work will be necessary to deliver clear answers on this topic.

\title{
Des cyclones et des hommes
}

\author{
Fabrice Chauvin et Jean-François Royer \\ Météo-France - Centre national de recherche météorologique (CNRM) \\ 42, avenue Gaspard-Coriolis - 31057 Toulouse Cedex 1
}

D e tout temps, les cyclones ont généré de nombreuses pertes en vies humaines ainsi que des dégâts matériels considérables. Les populations primitives avaient compris l'intérêt de s'installer en retrait des zones côtières sujettes au risque cyclonique. Avec le temps, il semble que cette précaution ait été oubliée et l'urbanisation croissante des côtes dans les tropiques rend les sociétés actuelles de plus en plus vulnérables aux cyclones tropicaux. Le nombre global annuel de phénomènes cycloniques est à peu près stable et se situe aux alentours de quatre-vingts systèmes. Mais les différents bassins océaniques connaissent des fluctuations naturelles de leur nombre de cyclones, liées à la variabilité du climat et du phénomène Enso (El Niño Southern Oscillation), en particulier.

La publication du $4^{\mathrm{e}}$ rapport du Groupe intergouvernemental d'experts sur l'évolution du climat (Giec) en 2007 (IPCC, 2007) est l'occasion de faire le point sur l'évolution attendue d'un certain nombre de phénomènes climatiques ayant une influence sur le bien-être des sociétés humaines. S'il ne fait désormais plus aucun doute que le climat moyen subira l'impact du réchauffement anthropique au cours du siècle qui vient de démarrer, il y a également de fortes présomptions pour que les événements extrêmes, au sens littéral du terme, subissent également des modulations. Malheureusement, la modification de ces évènements dans leur fréquence, leur durée, leur intensité ou leur localisation pourrait avoir des conséquences autrement plus graves que celles d'un réchauffement moyen de quelques degrés. Lors de la canicule de 2003 en Europe, on s'est clairement rendu compte que nos sociétés étaient très vulnérables à ces événements dits « extrêmes ». Parmi les

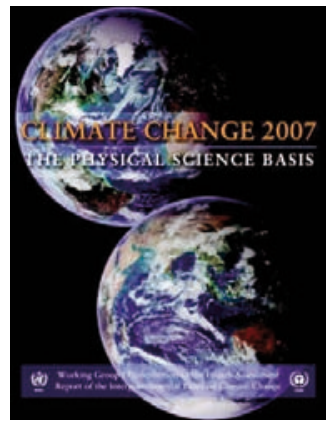

Couverture du $4^{\mathrm{e}}$ rapport du Giec.

événements extrêmes que notre planète peut générer, les cyclones tropicaux fascinent les hommes par leur puissance et leur beauté. Ils nous interpellent également par leur potentiel de destruction. La saison cyclonique de 2005, sur l'Atlantique, et particulièrement le cyclone Katrina qui a touché la Nouvelle-Orléans le 29 août de cette même année (Renaut, 2005), ont rappelé aux populations vivant sur le pourtour de la mer des Caraïbes qu'elles n'étaient pas à l'abri d'un phénomène majeur. Dès lors, il semble judicieux de se demander dans quelle mesure l'évolution que connaît actuellement notre climat pourrait se traduire par des changements dans l'activité cyclonique des différents bassins océaniques concernés par ce risque.

L'article qui suit va tenter de présenter l'état des connaissances de la communauté scientifique concernant l'impact du réchauffement sur l'activité cyclonique globale. Comme pour tous les autres phénomènes climatiques, il convient de faire la distinction entre ce que l'on peut déduire des observations passées et les projections futures. Ces dernières ne peuvent être abordées que par le biais de la modélisation climatique, alors que le climat passé est généralement bien documenté par les observations. Nous verrons que, malheureusement, ces observations ne sont pas un gage de certitude quant à l'évolution récente de l'activité cyclonique. 


\section{Historique}

Aussi loin que l'on remonte dans le temps, les effets ravageurs des cyclones tropicaux ont été ressentis par les sociétés humaines, et ce d'autant plus que cellesci tournaient leur activité vers les océans soumis à ces catastrophes naturelles. Le terme même d'ouragan, utilisé dans l'Atlantique nord et le Pacifique nord-est, aurait été donné par les explorateurs espagnols, qui l'auraient emprunté au langage des Indiens Tainos, pour qui huracan qualifiait un esprit démoniaque. Ceux-ci l'auraient eux-mêmes emprunté aux Mayas, chez qui Huraken était le dieu des tempêtes. Kerry Emanuel (2005a), dans son superbe livre dédié aux cyclones, Divine Wind, raconte la légende Taino, selon laquelle la naissance de ces vents serait due à la jalousie du dieu Guacar envers son frère Yucaju qui avait créé le Soleil et la Lune. Celui qui devait par la suite se faire appeler Jurakan, le dieu de la destruction, créa donc des vents puissants pour déchirer la Terre. Emanuel rapporte également que les Tainos pourraient avoir découvert le caractère rotationnel des ouragans bien avant que des moyens autres que visuels soient mis en œuvre pour le prouver. Toujours est-il que des figurations du dieu Huracan le représentent sous la forme d'un tourbillon. Néanmoins, cette représentation aurait pu être également inspirée des tornades ou des trombes, dont le caractère tourbillonnant est nettement plus facile à observer pour un être humain.

L'origine du terme typhon semble plus difficile à déterminer, mais il est très probable qu'il découle du terme chinois jufeng, c'est-à-dire « vent effrayant ou venant des quatre directions », que l'on retrouve dans la littérature du $\mathrm{v}^{\mathrm{e}}$ siècle.

\section{恳 $=$ 風 + 具}

Le terme typhon vient probablement du mot chinois jufeng qui est composé de l'élément substantif " vent ", feng (à gauche du signe +) et de l'élément phonétique ju (à droite du signe + ).

(Voir site Internet

www.oceanography.lsu.edu/faculty/liu/paleoecology web/index files/typhoons.pdf)

Pour faire l'historique complet des cyclones tropicaux, il faudrait y consacrer plusieurs livres, mais notre ami Kerry nous a gratifiés d'un résumé déjà suffisamment étayé pour pouvoir briller en société.

On sait donc que les sociétés primitives ont été concernées par ce phénomène, dont les interférences avec l'histoire ne manquent pas. Au cours du XIII ${ }^{\mathrm{e}}$ siècle,
Kubilai Khan, un empereur mongol descendant de Genghis Khan, tenta par deux fois d'envahir le Japon. À chacune de ses tentatives, sa flotte de plusieurs milliers de navires fut réduite en pièces par un typhon (en 1274 et 1281). Les Japonais ont cru voir là l'action d'un « vent divin » (kamikaze en japonais) qui aurait protégé leur indépendance. Quelques siècles plus tard et dans les Caraïbes, Christophe Colomb a perdu une partie de sa flotte, même si le phénomène qui lui a inspiré ce qu'on s'accorde à considérer comme le premier compte rendu européen sur les cyclones, pourrait n'avoir été qu'une trombe marine. À peine un siècle plus tard, en 1565, la France perdait la Floride au profit de l'Espagne après une rencontre malheureuse de sa flotte avec un cyclone. Les exemples de collisions entre l'histoire humaine et celle des cyclones ne manquent pas ; mais, même sans infléchir

Maison endommagée par l'ouragan Dennis le 18 juillet 2005 en Ontario, au Canada. l'histoire, les dégâts causés par ceux-ci ont toujours été énormes (photo ci-dessous). Pour ne parler que de l'Atlantique, le phénomène le plus meurtrier fut sans aucun doute le "Grand Cyclone » de 1780. Deuxième d'une série de trois cyclones qui se succédèrent sur les Caraïbes, il ravagea les côtes de la Barbade aux Bermudes, faisant plus de 22000 morts. En 1900, le cyclone de Galveston (Texas) fit entre 8000 et 12000 morts, détruisant la majeure partie de la première ville du Texas. Plus récemment, en 1974, le cyclone Fifi était le troisième cyclone le plus meurtrier de l'histoire de l'Atlantique, avec 10000 morts, avant d'être détrôné par Mitch en 1998, qui a fait 11000 morts.

Cette sombre liste de taux de mortalité dans l'Atlantique ouest n'est malheureusement qu'une " broutille », comparée aux 300000 à 500000 morts du cyclone qui toucha les côtes de ce qui s'appelait alors le Pakistan Est (Bangladesh actuel), en novembre 1970. Tout en étant le plus

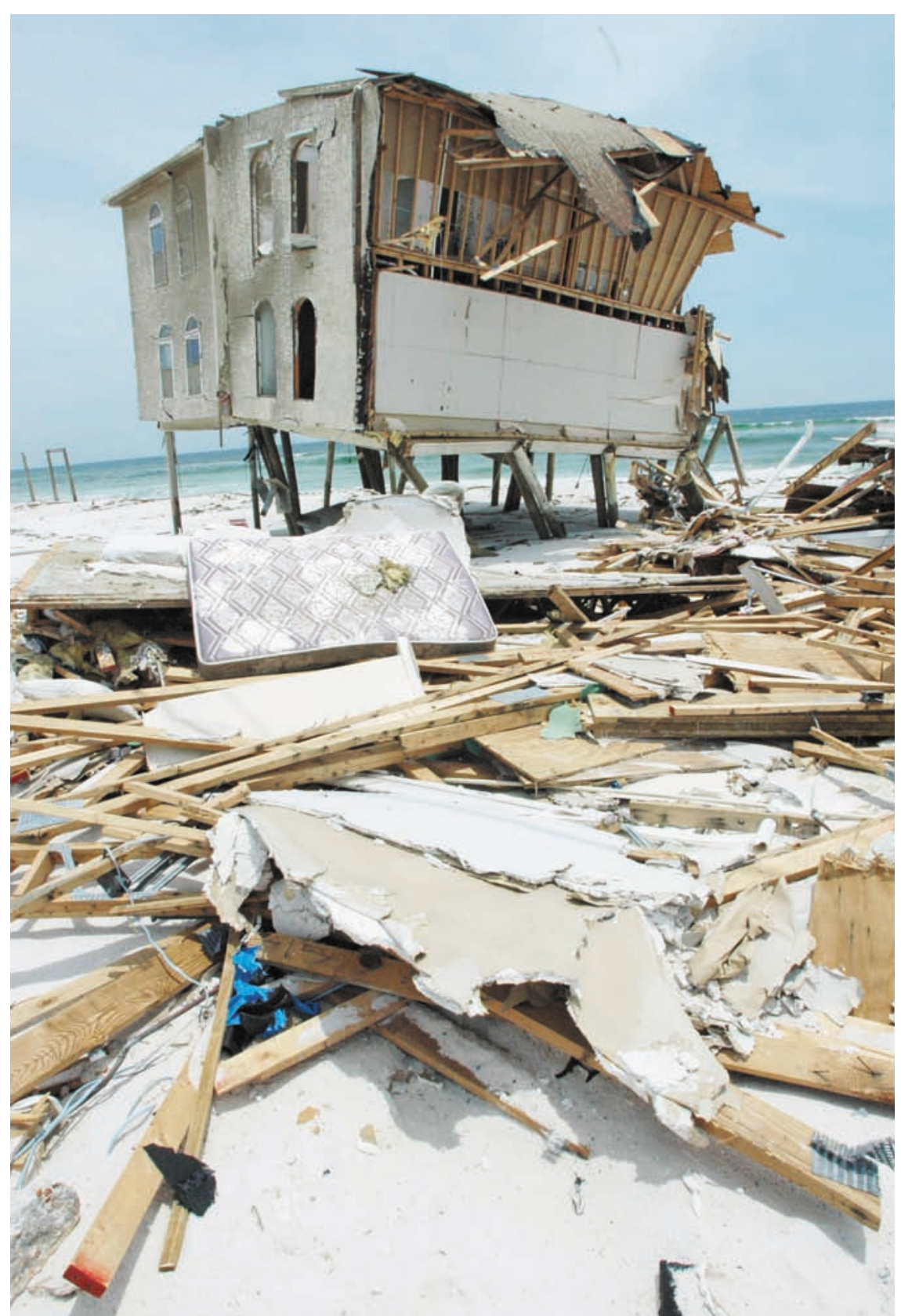


meurtrier de l'histoire des cyclones, cet événement n'est malheureusement pas isolé à cet endroit de la planète, où l'embouchure du Gange favorise la progression des marées de tempêtes si meurtrières. L'histoire cyclonique de cette région se chiffre en centaines de milliers de morts, soit plus de cent fois celle des Caraïbes.

Bien entendu, l'histoire des cyclones ne s'arrête pas à ces quelques exemples particulièrement marquants. Les noms de Hugo (1989), Andrew (1992), Floyd (1999) et, bien entendu, Katrina (2005) figurent parmi la liste des cyclones malheureusement célèbres de ces dernières années. Curieusement, hormis Mitch, ces cyclones sont loin d'engendrer les records en pertes humaines des cyclones des siècles précédents. En revanche, les coûts matériels occasionnés sont souvent les plus importants, à cause de l'évolution des prix, bien sûr, mais également à cause de la densification des régions côtières et de l'augmentation des biens assurés. Mais souvent, l'histoire même du phénomène ainsi que sa chronologie suffisent à expliquer l'ampleur des dégâts causés. À titre d'exemple, Katrina a détrôné Andrew en termes de coût, avec une facture de 80 milliards de dollars, alors qu'Andrew n'avait coûté « que » 30 milliards, treize ans plus tôt. Mais, si ce même cyclone avait atterri $100 \mathrm{~km}$ ou $200 \mathrm{~km}$ plus à l'est ou plus à l'ouest, personne ne s'en souviendrait aujourd'hui. On s'aperçoit ainsi qu'il est difficile de relater l'histoire des cyclones sans s'intéresser à l'histoire de chacun d'eux.

\section{Généralités}

\section{Représentation conceptuelle des cyclones}

Nous n'entrerons pas dans une explication approfondie des mécanismes en jeu dans les cyclones puisque cela n'est pas l'objet du présent article, mais nous mentionnerons tout de même quelques processus généraux qui caractérisent un cyclone tropical. Le lecteur désireux de creuser ce point peut consulter un article de référence (Roux et Viltard, 1997) publié dans cette même revue.

Au premier abord, on peut considérer un cyclone comme un gigantesque « vortex » de circulation cyclonique qui s'étend sur presque toute la profondeur de la troposphère en un tube de vents dont l'intensité, maximale en basse couche (au-dessus de la couche limite), décroît avec l'altitude. Pour respecter les lois de la dynamique, au sommet du cyclone, cette circulation s'inverse et devient anticyclonique. À ce vortex est associée une dépression centrale en surface, surmontée d'une anomalie de température dans les niveaux les plus hauts de la troposphère. Celle-ci est produite par les précipitations, qui libèrent la chaleur latente issue de l'évaporation de l'océan. La circulation tangentielle primaire perdure tant qu'elle est équilibrée par une anomalie chaude, via l'équilibre du vent thermique ; elle est érodée par le frottement de surface et la dissipation turbulente d'altitude. Une circulation radiale secondaire existe sur toute la profondeur de la troposphère, convergente en basse couche et divergente au sommet du cyclone. Cette circulation secondaire est essentielle à l'équilibre du cyclone en permettant l'introduction d'énergie dans son cœur par les effets du frottement exercé par le flux entrant sur la surface océanique. La branche ascendante de cette circulation secondaire est matérialisée par le mur de l'œil et les bandes externes qui s'enroulent en spirale autour, c'est-à-dire la région où la convection est intense. Les branches descendantes de cette circulation radiale sont constituées, d'une part, par la subsidence de grande échelle de la masse d'air environnant le cyclone et, d'autre part, par des mouvements subsidents au cœur de l'œil (ce qui en fait d'ailleurs une zone peu nuageuse).

Tout ce qui a été appris jusqu'à présent sur les mécanismes de formation des cyclones ne permet pas, actuellement, de comprendre en détail la formation de tels monstres. Bien entendu, on connaît les ingrédients de la recette qui permet de constituer un beau cyclone ; on sait également qu'il faut un tourbillon initial pour faire « monter la sauce "». Mais, ce qui fait que la recette est réussie ou pas, la petite touche du cordon bleu en quelque sorte, on ne le comprend pas encore. C'est par un savant dosage entre la convection et son inhibition que les cyclones peuvent se développer sur des océans. On considère, dans notre climat actuel, que les températures de la mer doivent dépasser $26{ }^{\circ} \mathrm{C}$ sur une profondeur de 60 mètres pour fournir suffisamment d'énergie au système. On verra plus loin que ce seuil n'a pas, en lui-même, de réalité physique absolue, mais permet de bien décrire les zones actuelles de cyclogenèse (la cyclogenèse est, étymologiquement, le mécanisme général de formation des phénomènes tourbillon- naires, mais on le restreindra ici à la seule formation des cyclones tropicaux). La convection est nécessaire à la formation d'un cyclone car elle permet l'humidification de l'atmosphère basse et moyenne (par évaporation des précipitations) et le réchauffement des niveaux plus élevés (par condensation de la vapeur d'eau ascendante). Elle n'est cependant pas suffisante pour produire un cyclone. L'organisation de la convection en cyclone nécessite d'introduire du tourbillon dans le système, c'est-à-dire du potentiel à mettre en œuvre un mouvement circulaire dans une région donnée. Ce tourbillon peut être fourni par des vortical hot towers (tours convectives de petite taille horizontale mais qui se développent à travers toute la troposphère) que l'on rencontre dans les amas convectifs océaniques isolés ou les vortex cycloniques de moyenne échelle. Mais il peut également provenir de perturbations dynamiques de type ondes d'est, de fluctuations en bordure d'une dépression de mousson ou de l'interaction entre la circulation des latitudes moyennes et la bande tropicale (Roux et Viltard, 1997). Les conditions de déclenchement de la cyclogenèse ont fait l'objet de nombreuses études et la question ne semble pas encore tranchée (Xu et Emanuel, 1989). L'instabilité convective de l'environnement ne semble pas être une condition nécessaire pour l'existence d'un cyclone. Sur océan, le profil vertical est plutôt neutre. En revanche, le déclenchement ne peut probablement pas avoir lieu si le profil vertical n'est pas instable. En tout cas, une condition vraiment nécessaire au déclenchement et au maintien des cyclones est qu'il existe un échange de chaleur entre l'atmosphère et l'océan sous-jacent. Cet échange est, en général, assuré par le frottement du vent radial de surface (Emanuel, 1986).

Si les conditions de déclenchement restent encore assez obscures, on connaît bien la physique du phénomène dans sa phase mature. Le modèle conceptuel le plus popularisé consiste à envisager les cyclones comme de formidables machines thermiques. Kerry Emanuel les apparente à des moteurs de Carnot dont l'efficacité est optimale (Emanuel, 1986).

Ce cycle a déjà été très bien décrit par Roux et Viltard (1997) dans cette même revue. On se bornera ici à en rappeler les grandes lignes. Roux et Viltard (1997) présentent, notamment, un schéma représentant le cycle de Carnot associé à un cyclone tropical à maturité (figure 1, page suivante). 


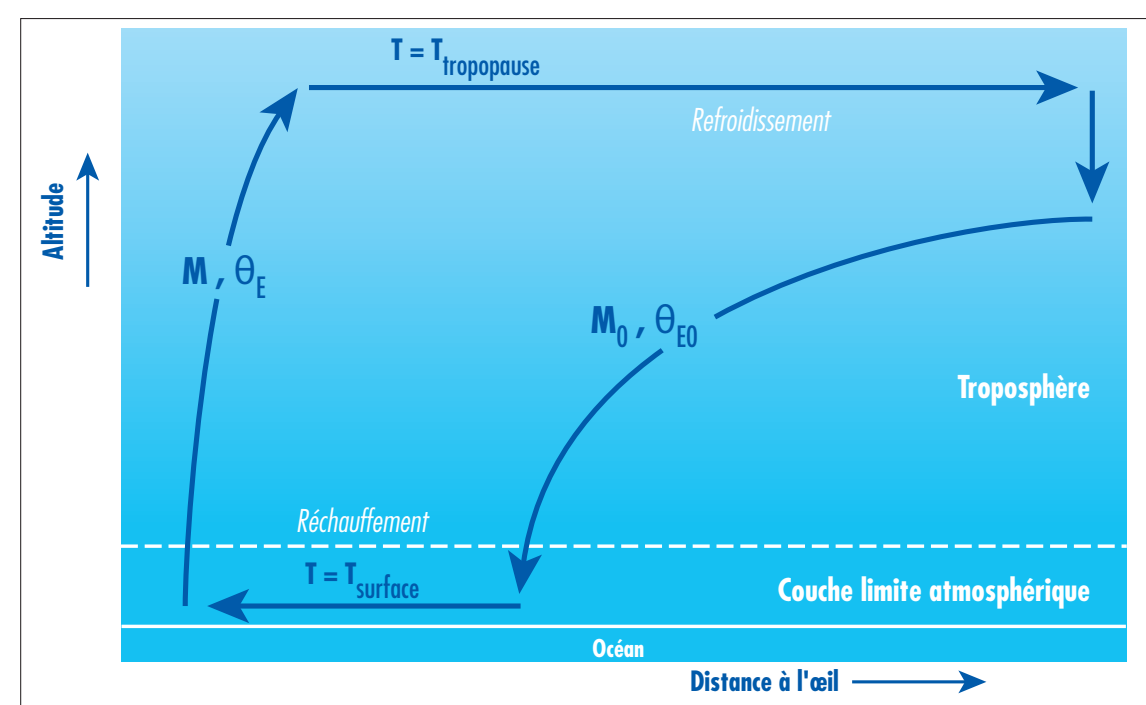

Le cycle énergétique de Carnot, tel que décrit par le physicien français au début $\mathrm{du} \mathrm{XIX}^{\mathrm{e}}$ siècle, comporte quatre phases qui contribuent à transformer de la chaleur en énergie mécanique. Si l'on considère un gaz contenu dans un cylindre fermé par un piston, les quatre phases se décomposent de la façon suivante (figure 2) :

- Phase 1 : expansion isotherme. Le gaz reçoit de la chaleur d'une source extérieure dont la température est constante. L'apport de chaleur est compensé par l'expansion du gaz, c'est-à-dire l'augmentation de son volume et la diminution de sa pression. Cette étape est isotherme car la température de la source de chaleur est constante.

- Phase 2 : expansion adiabatique. Lorsque la source de chaleur est retirée, le gaz continue son expansion, mais cette fois, la transformation est adiabatique car il n'y a plus d'échange de chaleur avec l'extérieur. Sa pression baisse ainsi que sa température.

- Phase 3 : compression isotherme. C'est l'inverse de la phase 1 : le gaz est mis en contact avec une source froide et perd de la chaleur. Il subit ainsi une compression isotherme

- Phase 4 : compression adiabatique. Après suppression de la source froide, le gaz continue de se comprimer et sa température augmente jusqu'à celle du début de cycle.
Ce schéma conceptuel, à la base de tous les moteurs mécaniques, représente bien la conversion des apports de chaleur (sources chaudes et froides) en énergie mécanique (le mouvement du piston). Le rendement du moteur est le rapport entre le travail produit et la chaleur. Carnot a montré que celui-ci était proportionnel à la différence entre la température de la source chaude $\left(\mathrm{T}_{\mathrm{c}}\right)$ et celle de la source froide $\left(\mathrm{T}_{\mathrm{f}}\right):\left(\mathrm{T}_{\mathrm{c}}-\mathrm{T}_{\mathrm{f}}\right) / \mathrm{T}_{\mathrm{c}}$.

Les quatre phases intervenant dans le moteur de Carnot se retrouvent dans le schéma conceptuel d'Emanuel (1986). La phase d'expansion isotherme (phase 1) correspond au parcours des particules de l'extérieur du cyclone vers l'intérieur de l'œil, où la source de chaleur est l'océan. Cette phase est bien associée à une forte baisse de la pression atmosphérique, comme suggéré par la théorie. Lorsque la particule d'air atteint les murs de l'œil, elle subit une forte ascendance qui peut être considérée comme adiabatique, même si des conversions de chaleur latente en chaleur sensible ont lieu au cours de cette phase (phase 2). Cette ascendance ne s'arrête qu'au niveau d'équilibre thermique de l'air, où celui-ci s'étale horizontalement, dans un mouvement divergent et anticyclonique. Cet équilibre thermique est atteint à la tropopause, au-dessus de laquelle la flottabilité devient négative à

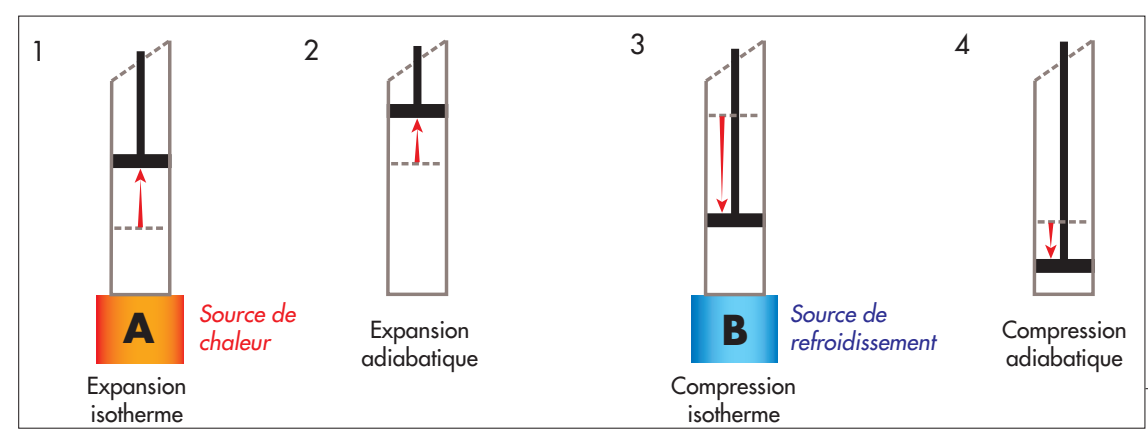

Figure 1 - Cycle de Carnot associé à un cyclone tropical à maturité. L'air entre dans la circulation cyclonique avec un moment angulaire absolu par unité de masse $\mathrm{M}_{0}$ et une température potentielle équivalente $\theta_{\mathrm{E} 0}$. II se dirige vers le centre du cyclone dans la couche limite avec une température constante $\mathrm{T}_{\text {surface, }}$ perdant du moment angulaire et gagnant de l'entropie humide par frottement sur la surface océanique. L'air subit une ascendance au voisinage du centre et s'en éloigne ensuite en haute troposphère, en maintenant constants son moment angulaire $M$ et sa température potentielle équivalente $\theta_{\mathrm{E} 0}$. À grande distance du centre, la température potentielle équivalente diminue par refroidissement radiatif vers l'espace et le moment angulaire augmente par interaction avec l'environnement. (Roux et Viltard, 1997)

cause du gradient vertical de température positif dans la stratosphère. Ainsi, en perdant de la chaleur par rayonnement électromagnétique, l'air subside de telle sorte que sa température reste constante (phase 3). Enfin, le retour à l'état initial se fait par compression adiabatique, car la chaleur que la particule perd par mélange avec son environnement est compensée par la perte de l'humidité du système par les pluies (phase 4). L'eau évacuée qu'il aurait fallu évaporer à mesure de la descente de la particule permet d'économiser l'énergie nécessaire à cette évaporation ; celle-ci est ainsi réinjectée dans le système.

Ce qui différencie ce moteur de celui de Carnot est que l'énergie mécanique générée par le système, à savoir les vents, est en partie réintroduite sous forme de chaleur par frottement avec la surface, optimisant d'autant le rendement du moteur. L'énergie acquise (essentiellement sous forme de chaleur latente) par frottement à la surface de l'océan est transformée en chaleur sensible par la condensation dans le mur de l'œil. Ce surplus de chaleur sensible entretient le flux ascendant. La subsidence compensatoire qui se produit dans l'œil produit un réchauffement d'altitude par compression adiabatique. Et pour respecter l'équilibre du vent thermique, la circulation tangentielle est accélérée, ce qui augmente l'énergie cinétique dissipée en surface. À l'équilibre, les énergies acquises et dissipées sont égales.

Ce modèle conceptuel a l'avantage de produire une représentation réaliste $d u$ cyclone en phase mature. Il a démontré, dans un certain nombre d'études théoriques, sa capacité à représenter de manière réaliste les caractéristiques du cyclone. De plus, il permet de déterminer une limite théorique à l'intensité des cyclones qu'aucun phénomène réel n'a 


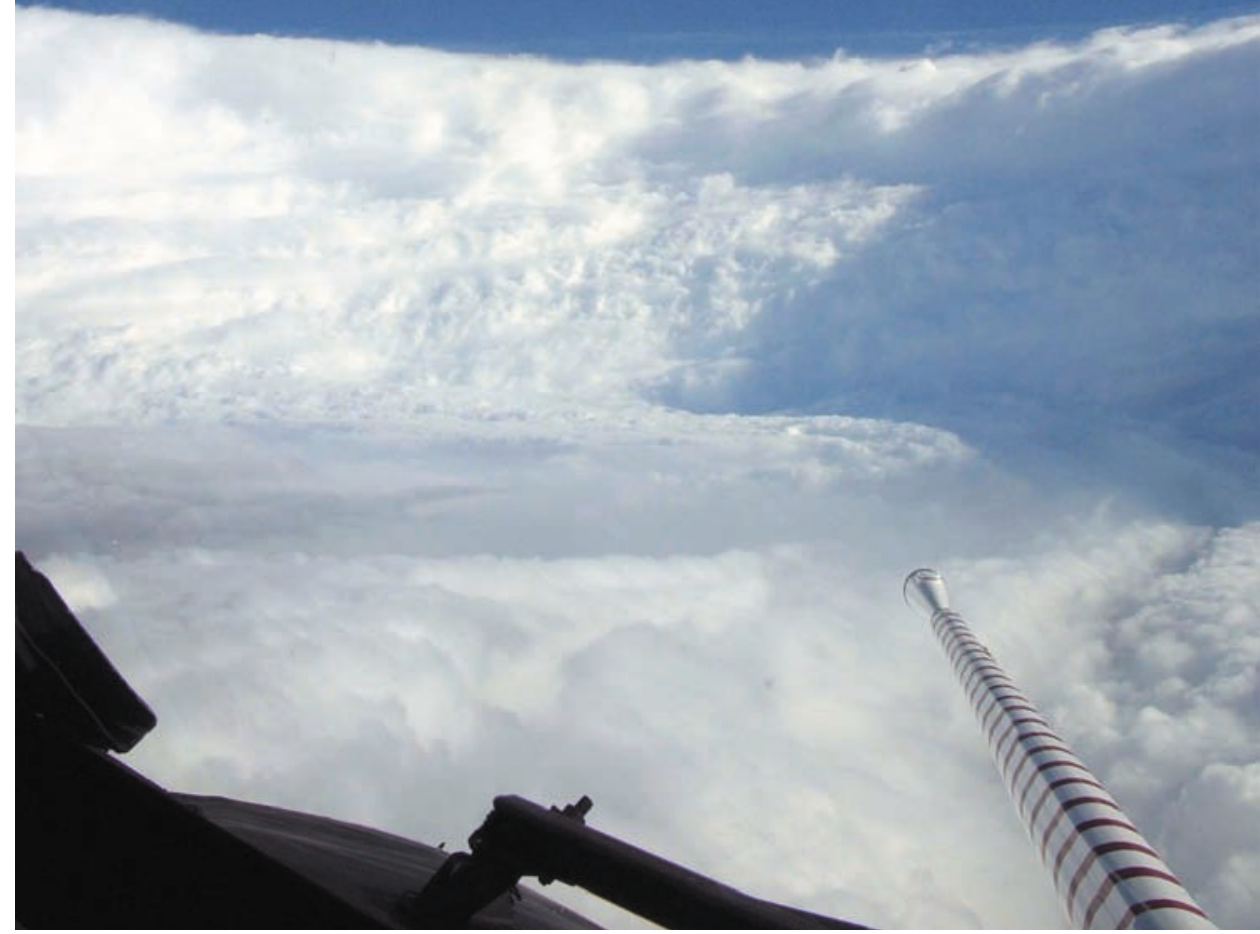

Le mur de l'œil du cyclone Katrina photographié depuis I'avion P3 " chasseur de cyclone " de la NOAA le 28 août 2005. (C NOAA)

encore jamais dépassée, et la majorité des systèmes est effectivement restée bien en deçà de cette limite (Emanuel, 1988). Enfin, nous avons présenté les cyclones comme des objets à symétrie circulaire, mais dans la réalité, celle-ci est très imparfaite. Il faut introduire des éléments de circulation générale pour expliquer pourquoi, dans l'hémisphère nord (respectivement sud) le vent est maximal dans la partie nord-est (respectivement sud-est) du cyclone. En effet, les vents dominants dans les tropiques venant de l'est, ceux-ci se conjuguent avec le système pour renforcer sa partie d'est « au vent ». Ainsi, dans l'hémisphère nord, le système tournant dans le sens inverse des aiguilles d'une montre, c'est le quart nord-est qui est renforcé. Le même raisonnement s'applique pour l'hémisphère sud dans lequel la rotation se fait dans le sens des aiguilles d'une montre. Mais c'est par les processus de moyenne échelle que les plus fortes discontinuités se développent. La redistribution du tourbillon potentiel au sein du système, le cycle de remplacement des murs de l'œil (ou anneaux convectifs), l'interaction avec des systèmes synoptiques de moyennes latitudes, la répartition de l'humidité dans la moitié la plus basse de la troposphère, etc., sont autant de possibilités d'asymétrie pour les cyclones. Si l'on y ajoute l'effet du gradient méridien du tourbillon planétaire, on voit bien que le modèle conceptuel axisymétrique trouve rapidement ses limites. Alors que les modèles de mésoéchelle peuvent produire une représentation complète des cyclones, nous verrons plus loin que les modèles de circulation générale sont cependant capables de représenter une grande partie de leurs caractéristiques malgré des résolutions qui ne permettent pas de résoudre l'ensemble de leurs composantes. C'est probablement dans cette direction que les recherches des prochaines années vont progresser dans la modélisation des cyclones.

\section{Observations}

L'observation des cyclones est une tâche difficile et s'est affinée au cours du temps, à mesure que les moyens de mesure se sont développés. Au cours du $\mathrm{XX}^{\mathrm{e}}$ siècle, un comptage systématique des cyclones a été entrepris sur un certain nombre de bassins océaniques, dont l'Atlantique a été le plus documenté. Mais ce comptage s'est fait avec de grandes disparités dans la qualité des mesures. Effectivement, avant l'avènement des mesures aéroportées et des satellites, les régions les mieux documentées étaient celles couvertes par le plus grand nombre de routes maritimes.

On peut distinguer trois grandes phases dans l'observation des cyclones, chacune ayant apporté une meilleure appréhension du nombre de cyclones ainsi que des caractéristiques des phénomènes observés (vent, pression, température...).

La première reposait entièrement sur l'observation visuelle et a duré du début des mesures jusqu'à la seconde guerre mondiale. N'étaient donc répertoriés que les cyclones touchant l'activité humaine. De plus, même si un système était observé, son intensité n'était pas forcément bien estimée, ce qui pouvait le reléguer au rang d'une simple tempête ou dépression tropicale. Si les cyclones qui atterrissaient (un terme qui désigne l'arrivée du cyclone sur les terres) étaient, en général, bien vus, les cyclones maritimes pouvaient passer complètement inaperçus pour peu qu'aucun navire n'ait eu à rencontrer leur trajectoire. Cependant, même les cyclones « terrestres » pouvaient parfois échapper aux observations, lorsque leur intensité était faible et qu'ils entraient sur le continent dans une région peu peuplée. Ainsi, avant l'introduction des avions dans les techniques d'observation, on pense que de nombreux cyclones n'ont pas été observés en tant que tels. On peut donc considérer qu'au cours de cette période, le nombre de cyclones a été systématiquement sousestimé, même s'il est difficile de préciser dans quelle mesure (Landsea, 2007).

Durant la seconde guerre mondiale, les vols de reconnaissance dans les cyclones, ont été initiés (photo ci-dessus) et, le 17 juillet 1944 , eut lieu le premier vol officiel dans un cyclone au-dessus de 1'Atlantique. Ce fut également l'époque où les mesures radar permirent d'observer que les précipitations étaient organisées en bandes spiralées autour de l'œil. Après la guerre, en 1946, des vols de reconnaissance audessus du Pacifique furent mis en place par l'armée américaine.

La troisième étape dans l'amélioration du système d'observation fût l'avènement des satellites météorologiques au cours des années 1960, qui ont permis une couverture globale et permanente de la planète (photo page suivante). En 
avril 1960, le satellite Tiros-1 put photographier un cyclone à 1500 kilomètres à l'est de l'Australie qui n'avait pas été repéré. L'étape suivante fut le lancement des satellites géostationnaires qui permettaient d'avoir une vue continue des zones de développement cyclonique. À titre d'exemple, le premier satellite Meteosat fut lancé le 23 novembre 1977. L'étape satellitaire fut primordiale dans l'observation des cyclones. En effet, bien que l'utilisation des satellites ne garantisse pas le décompte exact de cyclones dans tous les bassins océaniques (étant donné que ce décompte dépend de l'intensité du vent enregistré), elle apporte néanmoins la certitude de ne rater aucun phénomène.

\section{Bases de données}

Pour l'observation et la prévision des phénomènes cycloniques, l'océan global a été découpé en sept grands bassins, chacun étant sous la responsabilité d'un centre régional spécialisé ou Regional Specialized Meteorological Centre (RSMC). La liste des bassins océaniques et de leur RSMC est la suivante :

- Océan Indien nord (New Delhi, Inde),

- Océan Indien sud-ouest (La Réunion, France),

- Pacifique nord-ouest (Tokyo, Japon),

- Pacifique centre (Honolulu, ÉtatsUnis),

- Pacifique sud-ouest (Nadi, îles Fidji),

- Pacifique nord-est et Atlantique (Miami, États-Unis).

En plus des sept RSMC, on compte cinq centres d'alerte cyclonique ou Tropical Cyclone Warning Centre (TCWC), qui produisent des bulletins d'alerte dans des zones plus restreintes du globe :

- Océan Indien sud-est (Perth, Australie),

- Mer d'Arafura et golfe de Carpentrie (Darwin, Australie),

- Mer de Corail (Brisbane, Australie),

- Mer des Salomons et golfe de Papouasie (Port Moresby, Papouasie Nouvelle-Guinée),

- Mer de Tasmanie (Wellington, Nouvelle-Zélande).

Il n'existe pas, aujourd'hui, de base de données globale documentant l'ensemble des cyclones de la planète, mais chacun des bassins a sa propre base, dans laquelle les données collectées peuvent varier d'un bassin à l'autre.

Le 18 septembre 2006, sur l'Atlantique nord, l'image du satellite Goes 12 montre deux ouragans : Gordon, de classe 1 (au nord) et Hélène de classe 3 (au sud).
Plusieurs raisons peuvent expliquer cette absence d'une base globale. La première provient probablement des histoires différentes de chacun des grands centres ainsi que des bassins eux-mêmes. Il est clair que les bassins océaniques qui ont été le plus souvent parcourus par des navires de toutes sortes (seule source d'observation avant l'apparition des avions) ont une base plus étoffée que ceux dont les routes maritimes sont moins nombreuses. Ainsi, la base de données actuellement considérée comme la plus aboutie est celle qui couvre l'océan Atlantique. Elle concerne la période 1851-2006. On verra plus loins que, malgré sa relativement bonne qualité, cette base de données suscite de grandes empoignades scientifiques au sujet de la détection de l'effet du réchauffement climatique sur la cyclogenèse. En effet, comme toutes ses homologues, cette base de données souffre d'un manque d'homogénéité dû à l'évolution des techniques d'observations et d'estimation des intensités. Avec l'arrivée des données satellites et l'utilisation de la méthode de Dvorak pour l'estimation de l'intensité des cyclones (Dvorak, 1975 et 1984), l'estimation systématique est réalisée sur les mêmes bases pour tous les phénomènes.

De plus, l'observation satellite permet de s'assurer que tous les cyclones ont été observés. Une autre source de disparité entre les bases de données provient de la manière de mesurer les variables du cyclone. Par exemple, dans l'Atlantique, la mesure du « vent maximal moyen de surface », utilisé pour la classification cyclonique, est effectuée, par le National Hurricane Center (NHC), avec un pas de temps d'une minute, alors que l'Organisation météorologique mondiale (OMM) préconise dix minutes. Pour les autres bassins, les données ne remontent qu'au milieu des années 1940 et leur fiabilité ne peut être assurée que depuis les mesures satellites. Ainsi, la grande disparité des bases rend leur centralisation délicate, sachant que, pour utiliser celles-ci, des corrections sont souvent nécessaires pour tenir compte des différentes faiblesses d'observation au cours du $\mathrm{XX}^{\mathrm{e}}$ siècle.

Ce manque de base synthétique est un peu gênant lorsque l'on cherche à étudier les phénomènes cycloniques globaux. Des quelques études qui ont déjà été réalisées, on sait qu'il y a à peu près quatre-vingts tempêtes tropicales annuelles (Sugi et al., 2002). De ces quatre-vingts tempêtes, un peu moins de la moitié atteindront le stade de cyclone tropical. Il est bon de rappeler que l'on désigne par tempête tropicale les dépressions dont l'intensité des vents maxima en surface dépasse $17 \mathrm{~m} / \mathrm{s}$. Ce ne sont pas des cyclones tropicaux, mais un nom leur est attribué et elles entrent dans le décompte des phénomènes cycloniques. Au-delà de $33 \mathrm{~m} / \mathrm{s}$, la tempête devient officiellement un cyclone tropical. Sous ce nom générique se cache un phénomène appelé « ouragan » dans l'Atlantique et

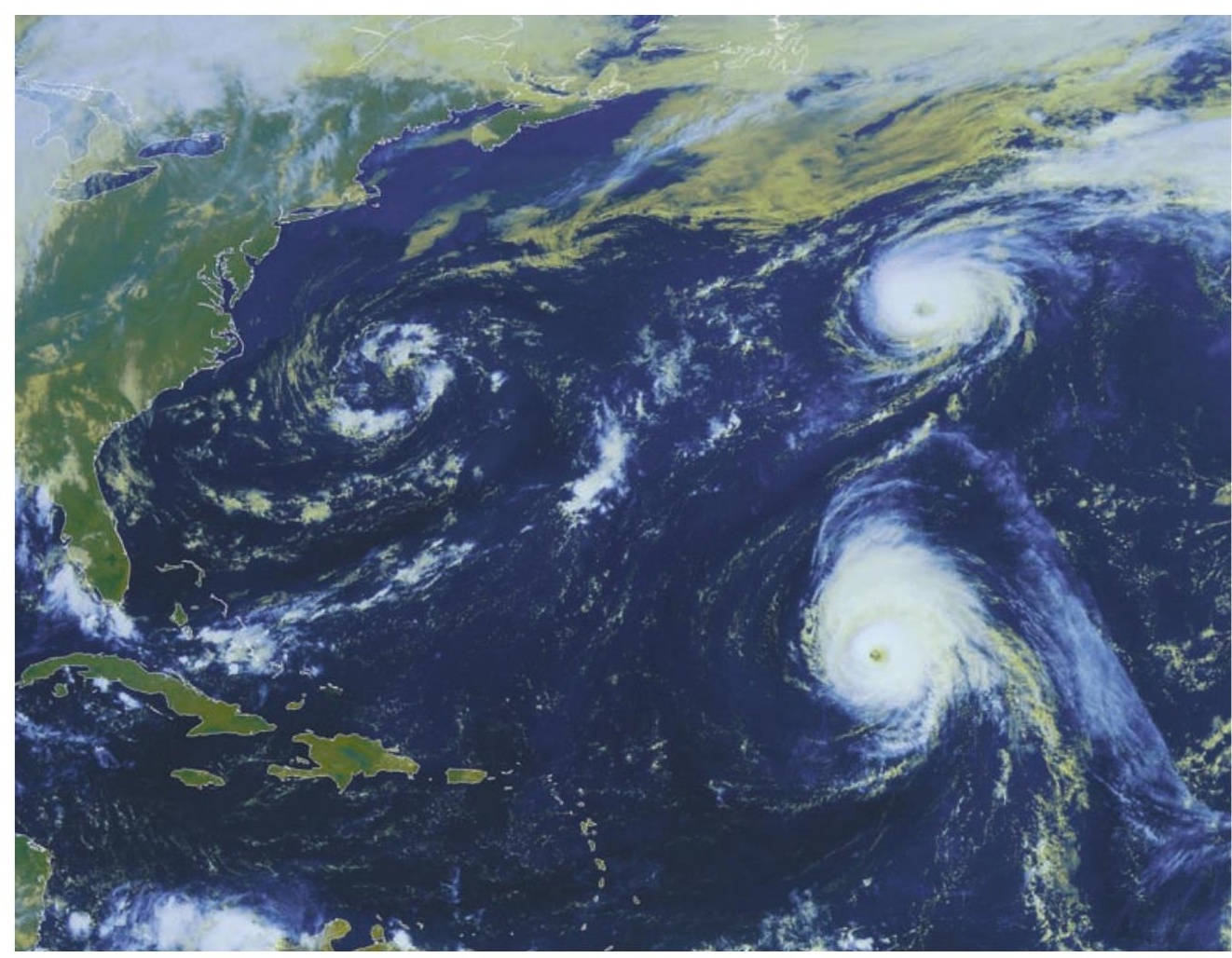


le Pacifique nord-est, " typhon », dans le Pacifique nord-ouest et tout simplement « cyclone tropical » ailleurs. L'attri-bution d'un nom à la tempête tropicale est très codifié et, là encore, les règles varient d'un bassin à l'autre. En revanche, dans tous les bassins, chaque phénomène reçoit bien un nom ou un numéro dès qu'il atteint le stade de tempête $(17 \mathrm{~m} / \mathrm{s})$. La plupart des bassins attribuent des prénoms aux cyclones pour permettre de mieux les référencer. Seuls l'océan Indien nord et le Pacifique nord-ouest utilisent des noms d'objets, de fleurs ou d'animaux. L'usage initial de donner un prénom uniquement féminin aux tempêtes tropicales a été modifié depuis 1975 dans le Pacifique sud-ouest, 1978 dans le Pacifique nord-est, l'Atlantique et le golfe du Mexique, au profit d'une alternance des prénoms masculins et féminins pour introduire plus de parité. Aujourd'hui, les noms sont attribués par des instances de l'OMM et cette parité s'applique dans tous les bassins concernés. Des listes alphabétiques sont préparées à l'avance pour chaque année et reviennent régulièrement. Lorsqu'un cyclone a marqué son époque par son caractère particulièrement dévastateur, son nom est retiré de la liste et remplacé par un autre. Ainsi, on ne reverra plus de Katrina ou d'Andrew en Atlantique.

\section{Quelques records}

Hormis les tristes records cités plus haut, et sans succomber à la tentation du sensationnalisme, on peut tout de même citer quelques records associés à des phénomènes cycloniques. Dans la littérature, de nombreux chiffres circulent, mais comme toujours en termes de records, ces chiffres se réfèrent parfois à un seul bassin. Nous ne citerons ici que des records globaux.

Le vent maximal jamais enregistré dans un cyclone est de $95 \mathrm{~m} / \mathrm{s}$ (typhon Nancy, septembre 1961), soit $342 \mathrm{~km} / \mathrm{h}$, la pression la plus basse de $870 \mathrm{hPa}$ (typhon Tip, octobre 1979), la marée de tempête la plus haute de $13 \mathrm{~m}$ (cyclone Mahina, 1899, en Australie) et la chute de pression la plus rapide de $100 \mathrm{hPa}$ en vingt-quatre heures (typhon Forrest, 1983). Les records de pluie associée aux cyclones varient en fonction du temps de cumul, mais ils sont en général observés à l'île de la Réunion (figure 3). Pour ne citer que deux exemples, le maximum de pluies enregistré en vingt-quatre heures $\mathrm{y}$ est de 1825 mm (cyclone Denise, jansur soixante-douze heures vient d'être battu par Gamède avec $3929 \mathrm{~mm}$ (février 2007). Les dimensions extrêmes des cyclones, mesurées comme le rayon de la zone de vents supérieurs à $17 \mathrm{~m} / \mathrm{s}$, ont été de $1100 \mathrm{~km}$ pour le plus grand (typhon Tip, 1979) à $50 \mathrm{~km}$ pour le plus petit (cyclone Tracy, décembre 1974, près de Darwin). Le cyclone à la plus grande longévité a duré 31 jours (typhon John, août-septembre 1994) alors que la plus longue distance parcourue a été de 13500 km (typhon Ophélia, novembredécembre 1960). Enfin, pour conclure cette liste de bêtes de cirque, le typhon Vamei, en Indonésie, est descendu jusqu'à $1^{\circ} 5 \mathrm{~N}$ en 2001 , ce qui représente la position la plus proche de l'équateur pour un cyclone tropical. Ces records communément admis sont évidemment susceptibles de changer à n'importe quel moment, soit parce qu'un nouveau record a été battu, soit parce que le cyclone détenant le record a été déclassifié. Ce pourrait être le cas du typhon Nancy dont on pense que les vents maximaux de $95 \mathrm{~m} / \mathrm{s}$ auraient pu être surestimés.

\section{Observations \\ à l'échelle paléoclimatique}

Outre l'observation en temps réel des cyclones tropicaux et la mise à jour des bases de données observées, il est un secteur émergent de l'étude des cyclones tropicaux qui se consacre à reconstruire l'histoire des cyclones au travers de l'observation de phénomènes géologiques (coraux, sédiments côtiers et lacustres, grottes) et biologiques (anneaux des arbres). En effet, le passage d'un cyclone laisse des traces en bordure de mer, en déposant des sédiments, en détruisant les barrières de coraux des îles touchées ou encore en changeant la composition en oxygène des pluies produites sur la région. En multipliant les sites d'étude de ces données paléoclimatiques, on espère reconstruire l'histoire des cyclones tropicaux sur des siècles. On peut notamment reconstruire les évolutions vier 1966), alors que le record de cumul

conjointes de l'activité cyclonique et des différents modes de variabilité climatique naturelle (Enso, Atlantic Multidecadal Oscillation...). Cette branche, assez jeune, des études paléoclimatiques porte le nom peu raffiné de « paléotempêtologie »(Liu, 2004). Cette discipline devrait permettre, dans l'avenir, de se faire une idée de la variabilité de l'activité cyclonique au cours des derniers siècles ou millénaires, dans des climats qui étaient très différents du nôtre. On entrevoit bien ce que cette discipline pourrait apporter au débat sur le changement climatique et ses conséquences en replaçant les changements futurs de l'activité cyclonique dans sa chronologie complète. Pour l'instant, étant donné la jeunesse de la discipline et le nombre limité de sites de mesures, il est évident que l'on ne peut accéder qu'à une vision qualitative de l'activité cyclonique passée.

\section{Modélisation climatique}

Dans cet article, nous entendons traiter de l'aspect climatique de la modélisation des cyclones, c'est-à-dire la manière dont ceux-ci sont représentés dans des Modèles de circulation générale (MCG). Ces derniers ne sont, en général, que des déclinaisons des modèles de prévision numérique utilisés opérationnellement, dont ils partagent au moins les équations d'évolution des variables thermodynamiques. Alors que les modèles opérationnels sont initialisés à l'aide des observations de l'atmosphère, les MCG sont forcés par des conditions de surface (températures de surface de la mer) ou atmosphériques globales (gaz à effet de serre, aérosols, volcanisme...). Nous n'aborderons pas les approches plus académiques d'étude des cyclones par des modèles plus fins mais à aire limitée. Si la résolution atteinte par ces derniers est très grande et permet une représentation très détaillée des phénomènes, il est difficile de les utiliser dans des études de changement climatique, pour des raisons de coût, mais aussi de conditions aux limites du domaine étudié. Ces modèles

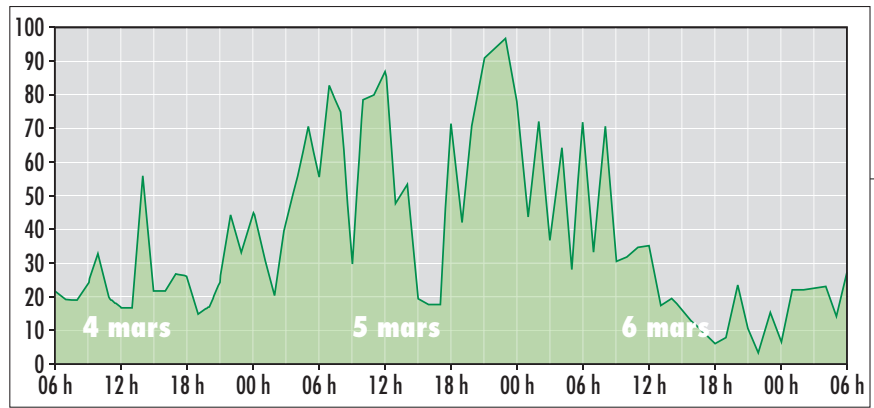

Figure 3 - Évolution des cumuls horaires de précipitations en mm à Commerson sur l'île de la Réunion, du 4 au 6 mars 2006, lors du passage de la tempête tropicale modérée Diwa. A Commerson, en trois jours, on a mesuré $2720 \mathrm{~mm}$. 
permettent d'étudier les changements de caractéristiques des cyclones dans un climat réchauffé, mais n'abordent généralement pas les questions de changement du nombre des phénomènes. En effet, les interactions globales du climat ne peuvent pas, par définition, être prises en compte dans ce type de simulation ou alors seulement par le biais des conditions aux limites imposées par l'analyse ou le modèle parent, de résolution plus grossière. De plus, se pose, dans ce cas, le problème de la trajectoire qui peut démarrer ou finir en dehors des limites du modèle à aire limité. La technologie du modèle avec grille « basculée/étirée » (dans lequel, la grille est déformée de manière à augmenter la résolution autour du pôle d'intérêt), dont le Centre national de recherche météorologique de MétéoFrance (CNRM) s'est fait une spécialité, peut être une alternative intéressante puisque le même modèle est utilisé pour représenter l'évolution de l'atmosphère globale et régionale, avec une transition continue entre les deux approches. Cette technologie a déjà été utilisée pour l'étude des cyclones au sein de notre équipe (Chauvin et al., 2006).

Lorsque l'on aborde le problème de la modélisation des cyclones tropicaux dans les modèles de climat, le premier écueil que l'on rencontre est lié à la taille du phénomène. Dans les simulations utilisées classiquement dans les études du climat, notamment dans les scénarios climatiques qui ont été réalisés pour le $4^{\mathrm{e}}$ rapport du Giec, la taille de la maille est généralement de l'ordre de $300 \times 300 \mathrm{~km}^{2}$, soit à peu près 100 fois la taille de l'œil d'un cyclone moyen. Autant dire que, dans ces simulations, ce qui pourrait se rapprocher le plus d'un cyclone n'est qu'une vaste et morne dépression tropicale, et les vents entourant le minimum de pression ressemblent plutôt à de douces brises. Bien sûr, dans des simulations plus courtes, on peut augmenter la résolution de telle sorte que le phénomène puisse être représenté de manière réaliste, mais le temps de calcul et le besoin de stockage de données pour de telles configurations, utilisées pour plusieurs dizaines d'années de simulations, devient un sérieux problème. Mais on a déjà vu apparaître, dans la communauté scientifique, des simulations de dix ans avec un modèle dont la résolution est de $20 \times 20 \mathrm{~km}^{2}$ (simulations réalisées par une équipe japonaise sur le supercalculateur Earth Simulator : Oouchi et al., 2006). Les ressources mises en œuvre pour réali-

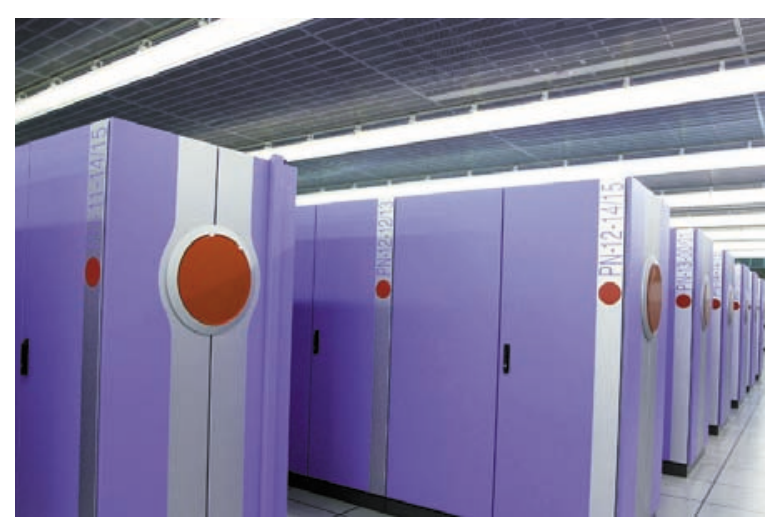

Le supercalculateur japonais, Earth Simulateur, sur lequel ont été réalisées des simulations de dix ans avec un modèle dont la résolution est de $20 \times 20 \mathrm{~km}^{2}$

ser ces expériences de deux fois dix ans rendent néanmoins l'exercice difficile à généraliser. Cependant, il ne fait aucun doute que les dix prochaines années verront la multiplication de ce genre de simulations et tout laboratoire qui se respecte se devra de montrer comment son modèle reproduit de jolis cyclones.

Le CNRM n'a pas à rougir de ses propres simulations puisque nous avons réalisé deux expériences de dix ans chacune avec une résolutions uniforme de 50 x $50 \mathrm{~km}^{2}$, ce qui nous place directement derrière les équipes japonaises en termes de résolution. De plus, pour des études localisées au bassin Atlantique, nous avons eu recours à la technologie de la grille basculée/étirée qui nous permet d'atteindre des résolutions de 50 x $50 \mathrm{~km}^{2}$ sur la zone d'intérêt pour le coût d'une simulation globale de résolution 2,5 fois plus grossière. Cela nous place en bonne position pour des études plus systématiques des cyclones dans un cadre climatologique.

Pour l'instant, nous n'en sommes pas encore là, et il faut se contenter, soit de courtes simulations à fine échelle, soit de longues simulations grossières. Dans les premières, on peut mettre en place des algorithmes de détection des cyclones et de construction des trajectoires. Dans les secondes, en revanche, cette méthodologie n'est plus adéquate, mais on peut, malgré tout, s'en sortir en étudiant les facteurs de grande échelle favorables à la cyclogenèse.

\section{Approche directe}

Comme nous l'avons indiqué ci-dessus, l'approche directe consiste à détecter directement les phénomènes cycloniques dans les simulations, à partir des variables du modèle, et à en construire les trajectoires. Pour cela, on met en place des algorithmes de détection et de naires qui n'auraient pas cyclone. La problématique pour la recherche des trajectoires cycloniques est tout à fait différente de celle des dépressions de moyennes latitudes. En effet, aux moyennes latitudes, on commence par rechercher, pour chaque jour de la simulation, tous les tourbillons, puis on cherche à construire des trajectoires en appariant ces tourbillons deux à deux d'un pas de temps à l'autre (Ayrault et Joly, 2000). La première étape est plutôt facile puisqu'il suffit de se donner un seuil d'intensité pour les tourbillons et de « scanner » les sorties du modèle. La seconde étape, n'est pas aussi simple qu'elle y paraît car, pour apparier deux tourbillons consécutifs, il faut choisir ceux qui se marient le mieux, parmi tous les couples possibles entre les deux échéances. Cela demande un algorithme un peu plus complexe faisant intervenir un critère de qualité des appariements. Pour la détection et le calcul de trajectoires des cyclones tropicaux, la problématique est inversée. La détection demande de fixer un certain nombre de critères pour être sûr de ne sélectionner que des tourbillons ayant toutes les caractéristiques de cyclones. Une fois cette étape réalisée, la question de l'appariement est nettement plus facile à résoudre, la liste des candidats ne laissant généralement que peu d'incertitude quant au choix des couples, en raison de la rareté de cyclones multiples (mais le cas peut parfois se rencontrer). Les différentes études qui ont eu recours à ce genre de programmes diffèrent légèrement dans les détails des critères imposés ; mais, globalement, pour détecter correctement un phénomène cyclonique dans une simulation climatique, il faut :

- un champ de tourbillon گ (c'est-à-dire rotationnel du vent horizontal) conséquent $\left(\zeta>\mathrm{S}_{\zeta}\right)$;

- un minimum de pression ;

- un cœur chaud, c'est-à-dire une anomalie de température, $\Delta \mathrm{T}$, plus forte en altitude qu'en basse couche $\left(\Delta \mathrm{T}>\mathrm{S}_{\Delta \mathrm{T}}\right)$; 
- des vents forts en basse couche $\left(\mathrm{V}_{850}>\right.$

$\mathrm{S}_{\mathrm{V} 850}$ et $\mathrm{V}_{850}>\mathrm{V}_{200}$ );

- une durée de vie suffisante ;

où $\Delta \mathrm{T}$ est l'anomalie de température entre le cyclone et son environnement (notions définies ci-dessous) et $\mathrm{V}_{850}$ et $\mathrm{V}_{200}$ sont le module du vent à $850 \mathrm{hPa}$ et $200 \mathrm{hPa}$ dans le cyclone.

Le calibrage de l'algorithme va consister à fixer les seuils $S_{\zeta}, S_{\Delta T}$ et $S_{V 850}$, qui vont déterminer le nombre de trajectoires détectées, ainsi qu'à définir le « cyclone » et son « environnement ». Dans le cas des simulations réalisées au CNRM avec un modèle ayant une maille de $50 \mathrm{~km}$, les seuils adoptés ont été choisis empiriquement, de telle sorte que le nombre de tempêtes obtenu corresponde à la climatologie sur les cinquante dernières années, et les valeurs des seuils obtenues sont les suivantes :

$$
\begin{aligned}
& \mathrm{S}_{\zeta}=1,4.10^{-4} \mathrm{~s}^{-1} \\
& \mathrm{~S}_{\Delta \mathrm{T}}=3{ }^{\circ} \mathrm{C} \\
& \mathrm{S}_{\mathrm{V} 850}=15 \mathrm{~ms}^{-1}
\end{aligned}
$$

Pour définir le cyclone, nous avons calculé pour chaque cyclone rencontré, un rayon de référence $(\mathrm{RdR})$ qui représente la distance entre le maximum de vent de basse couche et le centre. Ensuite, la zone qui détermine le cyclone a été définie comme le disque de rayon $2 * \mathrm{RdR}$ centré sur le centre du cyclone. Cette zone est extraite d'un disque de rayon $6 * \mathrm{RdR}$ qui représente l'environnement du cyclone. Ainsi, un rapport 9 existe entre la surface du cyclone et celle de son environnement. Le calcul de $\Delta \mathrm{T}$ est effectué sur la moyenne entre les couches comprises entre $700 \mathrm{hPa}$ et $300 \mathrm{hPa}$ (Chauvin et al., 2006).

Cette méthodologie est, bien entendu, loin d'être parfaite ; mais, actuellement, elle donne des résultats assez convaincants pour permettre de s'y fier. Elle donne notamment une répartition très réaliste des trajectoires sur le bassin Atlantique, malgré une tendance à surestimer le nombre de trajectoires au-dessus du golfe du Mexique. Elle présente surtout l'intérêt d'appréhender comment le modèle, soumis à des conditions de forçage particulières, telles que la concentration de $\mathrm{CO}_{2}$ associée à une anomalie de température de surface de la mer (TSM), module ses phénomènes cycloniques, sans avoir besoin de formuler des hypothèses supplémentaires sur la relation entre l'environnement climatique et l'activité cyclonique.

\section{Approche par la grande échelle (méthodes indirectes)}

Comme nous l'avons déjà signalé, la résolution insuffisante du modèle ne permet pas toujours d'employer la méthode directe. On peut néanmoins se faire une idée du changement dans la cyclogenèse par un moyen détourné qui consiste à regarder comment les conditions de grande échelle, favorables à la cyclogenèse, évoluent lorsque les conditions de forçage changent. Cette approche a été popularisée dès les années 1960, grâce aux travaux de William Gray (Gray, 1968), qui ont abouti à la mise au point d'un indice de cyclogenèse dépendant de la combinaison de considérations thermiques et dynamiques. Nous allons détailler cet indice, dont le nom anglais est Yearly Genesis Parameter (YGP), afin de montrer sa puissance, mais aussi ses limites pour l'étude du changement climatique.

Cet indice YGP est la somme de quatre paramètres saisonniers SGP (pour Seasonal Genesis Parameters), exprimés par le produit de deux potentiels, thermique et dynamique, chacun d'eux étant lui-même composé de trois facteurs. Ces facteurs sont calculés mensuellement ou par saison, mais traduisent un état moyen de l'atmosphère au cours de la saison cyclonique.

\section{Le potentiel dynamique PD \\ C'est le produit :}

$\mathrm{PD}=(\zeta+5) \cdot|f| \cdot(\Delta \mathrm{V} / \Delta \mathrm{P}+3)^{-1}$, en $\mathrm{s}^{-1} \mathrm{~m}^{-1} \mathrm{hPa}$,

où $|f|$ est la valeur absolue du paramètre de Coriolis, qui dépend de la latitude ( $f=2 \Omega \sin \varphi$, où $\Omega=7,29.10^{-5} \mathrm{rad} / \mathrm{s}$ est la vitesse angulaire de rotation de la Terre et $\varphi$ la latitude), $\zeta$ est le tourbillon relatif à $950 \mathrm{hPa}$ pondéré par le signe de $\mathrm{f}$ pour tenir compte de l'hémisphère dans lequel est calculé l'indice, et $\Delta \mathrm{V} / \Delta \mathrm{P}$ le cisaillement vertical du vent horizontal entre 925 et $200 \mathrm{hPa}$.

Ce potentiel exprime ainsi que la cyclogenèse ne peut avoir lieu qu'à une distance respectable de l'équateur (où $\sin \varphi$ est nul), dans un environnement tourbillonnaire et peu cisaillé verticalement. Le dernier facteur traduit le caractère inhibant du cisaillement vertical du vent horizontal : celui-ci a un effet inhibiteur en empêchant le développement vertical du cyclone et en favorisant les échanges avec l'environnement par ventilation des niveaux intermédiaires du cyclone. $\mathrm{Ce}$ dernier critère se révèle être un facteur prépondérant sur l'océan Atlantique, alors que sur les autres bassins, d'autres facteurs vont dominer. La faible largeur de cet océan explique probablement qu'en présence de cisaillement, les ouragans n'ont pas le temps de se développer au cours de leur traversée du bassin. De plus, le cisaillement sur l'Atlantique est lui-même fortement modulé par l'Enso et la QBO (Quasi Biennale Oscillation), un phénomène stratosphérique. Rappelons enfin que le cisaillement vertical, s'il inhibe les cyclones, favorise, sur continent, la convection, les orages et les tornades.

\section{Le potentiel thermique PT}

C'est le produit :

$\mathrm{PT}=\mathrm{E} .(\Delta \theta / \Delta \mathrm{P}+5) \cdot \max ((\mathrm{RH}-40) / 30,1)$, en cal $\mathrm{cm}^{-2} \mathrm{~K} \mathrm{hPa}^{-1}$,

où $\mathrm{E}$ est le contenu thermique des soixante premiers mètres de l'océan, défini comme l'intégrale de l'excédent de température au-dessus de $26{ }^{\circ} \mathrm{C}$ sur cette profondeur, $\Delta \theta / \Delta \mathrm{P}$ est la différence de température potentielle entre la surface et $500 \mathrm{hPa}$ et RH est l'humidité relative entre $500 \mathrm{hPa}$ et $700 \mathrm{hPa}$.

Les constantes entières introduites dans les formules servent, compte tenu des unités dans lesquelles sont exprimées les variables, à assurer des valeurs non nulles aux différents facteurs, excepté le paramètre $f$.

Au final, le paramètre saisonnier complet de cyclogenèse est le produit des deux potentiels :

$\mathrm{SGP}=\mathrm{PD} . \mathrm{PT}$, en cal $\mathrm{K} \mathrm{s}^{-1} \mathrm{~cm}^{-3}$,

et s'exprime en nombre de cyclogenèses par carré de 5 degrés de côté, par vingt ans et par saison.

Pour obtenir le paramètre YGP, on effectue la somme des quatre paramètres saisonniers SGP.

Ce paramètre s'est avéré reproduire correctement la répartition des cyclogenèses dans les différents bassins au cours des cinquante dernières années, ce qui en fait un outil essentiel pour l'étude de la cyclogenèse, notamment de sa variabilité interannuelle ou décennale (cycles de périodes de l'ordre de dix ans). En effet, on peut relier les fluctuations de l'activité cyclonique à celles du YGP. Et bien entendu, à partir du moment où les conditions cycloniques peuvent changer 
avec les conditions de grande échelle, on est en droit de se demander quelle évolution le siècle actuel nous réserve.

\section{Évolution de l'activité cyclonique avec le réchauffement anthropique}

L'action de l'homme sur les cyclones, avant de se faire sentir par les effets du réchauffement anthropique, avait déjà été envisagée, au lendemain de la seconde guerre mondiale, pour essayer d'affaiblir l'intensité de ces monstres dévastateurs. Le 13 octobre 1947, dans le cadre du projet Cirrus (Willoughby et al., 1985), des particules d'iodure d'argent furent lâchées d'un avion au cœur d'un cyclone qui, malheureusement et sans lien apparent avec l'action intentée, infléchit sa course initiale pour aller frapper les côtes de la Georgie et de la Caroline du Sud. Les essais ultérieurs ont donc été dorénavant plus encadrés. Notamment, les expériences d'ensemencement ne furent désormais réalisées que dans des cyclones se trouvant loin des côtes et ne risquant pas de s'en approcher. L'idée sous-jacente à l'ensemencement est qu'en introduisant des noyaux de condensation à l'extérieur du mur de l'œil, on favorise la formation de nuages à partir de l'eau surfondue qui y séjourne. Le développement de nuage à l'extérieur du mur de l'œil pourrait ainsi priver celui-ci de son alimentation, l'affaiblissant ainsi au profit d'un nouveau mur plus large et moins puissant. Cette idée fit son chemin au cours des années 1950 , pour aboutir, en 1962, à la création du projet Stormfury (Willoughby et al., 1985). Ce projet s'est déroulé de 1962 à 1983, et de nombreux ensemencements ont été réalisés à cette occasion. Quelques résultats furent obtenus, mais la complexité du protocole opérationnel, le manque de financement et la relative accalmie des années 1970-1980 sur l'Atlantique eurent raison de cette expérience. D'autant que, au fur et à mesure que les connaissances avançaient sur la structure des cyclones, les bases scientifiques du projet s'étiolaient et les effets obtenus par l'ensemencement étaient remis en cause. En effet, l'eau surfondue, dans un cyclone, n'est pas si abondante qu'on le pensait, et le processus d'affaiblissement du mur interne de l'œil est un effet naturel qui s'accompagne de son remplacement progressif par un nouveau mur, constitué de bandes externes qui se structurent et s'intensifient lors de leur migration vers le centre du cyclone.
D'autres idées d'action pour réduire l'intensité des cyclones ont germé au cours des cinquante dernières années. On a pensé, entre autres, à utiliser des satellites pour bombarder le cyclone de micro-ondes ou à déverser un film chimique à la surface de l'océan pour isoler le cyclone de sa source de chaleur. Mais vu la puissance du phénomène, les ressources à mettre en jeu pour le modifier seraient énormes et les effets secondaires importants.

Finalement, aujourd'hui, l'homme se demande s'il n'est pas en train de réussir, par son action involontaire sur le climat, ce qu'il a vainement essayé de faire pendant des années. Ainsi, la question de l'influence du réchauffement des océans sur l'activité cyclonique pourrait devenir cruciale pour les sociétés à venir. De nombreuses populations ont de tout temps habité en bordure des bassins océaniques et sont donc vulnérables à l'activité cyclonique dans tous les bassins. Les Mayas avaient bien compris le problème puisqu'ils avaient construit leurs villes à l'intérieur des terres. Il semble que cette expérience ait été oubliée au fil des siècles et l'explosion démographique que connaissent les côtes américaines, mais également de nombreuses zones côtières de pays en développement soumises au risque cyclonique (Caraïbes, Philippines, Bangladesh, Inde...), laisse présager une vulnérabilité accrue des sociétés aux phénomènes cycloniques.

La première idée qui vient à l'esprit lorsque l'on envisage le réchauffement anthropique est que l'élévation des températures de surface de la mer (TSM) engendrera forcément une augmentation de l'activité cyclonique, plus d'énergie étant disponible pour les systèmes. Cette idée a fait son chemin depuis longtemps, notamment grâce au fameux seuil de $26{ }^{\circ} \mathrm{C}$ considéré comme nécessaire à la formation des cyclones et qui verrait son aire de jeu augmenter significativement dans un monde réchauffé.

Les premières études reposant sur l'indice YGP de Gray confirmaient bien cette dépendance, puisque le contenu thermique de l'océan était approché par l'écart de la TSM à $26^{\circ} \mathrm{C}$. Ainsi, par exemple, sur un océan initialement à $28{ }^{\circ} \mathrm{C}$ dans le climat présent et qui se réchauffe de $2{ }^{\circ} \mathrm{C}$ dans l'avenir, le contenu thermique serait doublé, et parlà même le paramètre de Gray. Ces évaluations pessimistes des prémices de scénarios climatiques ont été depuis revues largement, notamment grâce aux travaux effectués au CNRM (Royer et al., 1998), dans lesquels la remise en question du seuil fatidique de $26^{\circ} \mathrm{C}$ a joué un rôle essentiel. Si l'on remonte à l'origine de ce seuil, on trouve les travaux de Pàlmen (1948) qui commença à s'intéresser aux conditions favorables à la cyclogenèse. Parmi les facteurs favorables, il avait considéré que le profil vertical de température atmosphérique devait être instable pour favoriser le déclenchement du cyclone (condition qui n'est plus nécessaire par la suite). En cherchant à traduire cela à l'aide d'une entité facile à appréhender et en fixant l'humidité des basses couches à $85 \%$, il avait déterminé quelle devait être la température de surface pour que le profil soit instable jusqu'à une altitude d'au moins $10 \mathrm{~km}$. Celle-ci était la température de surface pour laquelle la différence entre la température à $300 \mathrm{hPa}$ et celle obtenue en montant adiabatiquement une particule depuis la surface soit négative. Le seuil de $26-27{ }^{\circ} \mathrm{C}$ s'est révélé délimiter la zone de cyclogenèse de manière suffisamment correcte pour être adopté. Depuis, les études effectuées sur ce seuil ont parfois tendance à oublier que cette température théorique dépend de l'humidité en surface et de la température au sommet de la troposphère. Le détail a son importance lorsque l'on sait que le réchauffement anthropique touchera autant la haute troposphère que les TSM. Ainsi, l'indice YGP de Gray, dans sa forme initiale, ne peut être utilisé pour des études de changement climatique, ce seuil de $26{ }^{\circ} \mathrm{C}$ n'étant plus valide dans un climat réchauffé. C'est ce qui a motivé l'approche de Royer et al. (1998). Partant du principe que les modèles pouvaient générer eux-même leurs propres conditions favorables à la cyclogenèse, le potentiel thermique y a été remplacé, dans sa globalité, par les précipitations convectives diagnostiquées dans le modèle, qui synthétisent à elles seules les contraintes de contenu thermique de l'océan, d'humidité et de profil vertical de température. Celles-ci sont, en effet, produites par le schéma de convection activé dans le modèle et dépendent donc de la température de surface et les profils d'humidité et de température de l'atmosphère. Il reste à faire l'hypothèse que la température de surface est un traceur du contenu thermique de l'océan pour accepter définitivement la précipitation convective comme critère thermique. Cette hypothèse est tout à fait acceptable aux échelles mensuelles ou saisonnières. Le nouvel indice obtenu, surnommé Convective Yearly Genesis Parameter (CYGP) s'est révélé plus propice à représenter les changements 
de la cyclogenèse dans une atmosphère réchauffée. Récemment, le calcul de cet indice a été effectué pour une grande partie des simulations produites par le Giec, dans le cadre de son $4^{\mathrm{e}}$ rapport. Les résultats seront abordés dans la suite de ce chapitre.

Toute cette discussion n'est évidemment utile que lorsqu'on utilise l'approche indirecte pour estimer les changements dans l'activité cyclonique. Avec l'approche directe, le modèle produit ses propres cyclones et il n'y a plus qu'à les compter dans les simulations présentes et futures pour en déduire les changements.

\section{Ce qu'on peut dire des tendances passées}

Avant d'aborder les projections des scénarios pour le futur, il est important de regarder ce que le passé nous apprend sur l'évolution de l'activité cyclonique au cours du XX $\mathrm{X}^{\mathrm{e}}$ siècle. Comme nous l'avons signalé dans la partie consacrée aux observations, les bases de données sont trop hétérogènes, actuellement, pour en tirer des conclusions irréfutables. Néanmoins, la saison cyclonique 2005 sur l'océan Atlantique a marqué, par son caractère exceptionnel, un véri-

\begin{tabular}{|ccccc}
$\begin{array}{c}\text { Catégorie } \\
\text { Saffir- } \\
\text { Simpson }\end{array}$ & $\begin{array}{c}\text { Maximum } \\
\text { du vent } \\
(\mathrm{m} / \mathrm{s})\end{array}$ & $\begin{array}{c}\text { Pression } \\
\text { minimale } \\
(\mathrm{hPa})\end{array}$ & $\begin{array}{c}\text { Marée } \\
\text { de tempête } \\
(\mathrm{m})\end{array}$ & $\begin{array}{c}\text { Dégâts } \\
\text { relatifs } \\
(33 \mathrm{M} \$)\end{array}$ \\
\hline 1 & $33-42$ & $>980$ & $1-1,7$ & 1 \\
\hline 2 & $43-49$ & $979-965$ & $1,8-2,6$ & 10 \\
\hline 3 & $50-58$ & $964-945$ & $2,7-3,8$ & 50 \\
\hline 4 & $59-69$ & $944-920$ & $3,9-5,6$ & 250 \\
\hline 5 & $>69$ & $<920$ & $>5,6$ & 500 \\
\hline
\end{tabular}

Figure 4 - Échelle de Saffir-Simpson et caractéristiques associées.

table tournant dans l'étude de l'impact du réchauffement climatique sur l'activité cyclonique. Effectivement, cette année-là, de nombreux records cycloniques ont été battus. Le record de 1933 de vingt-et-un systèmes avec des vents maximaux dépassaent $17 \mathrm{~m} / \mathrm{s}$ a été pulvérisé, avec vingt-huit systèmes en 2005. Parmi les autres records battus, citons celui du nombre d'ouragans (quinze systèmes ont atteint l'intensité de cyclone tropical, le précédent record étant de douze en 1969); du nombre de cyclones de catégorie 5 sur l'échelle de Saffir-Simpson (cette échelle est reportée figure 4), parmi lesquels trois des six ouragans les plus forts jamais enregistrés dans le bassin Atlantique (quatre cyclones de catégorie 5 pour seulement deux en 1960 et 1961); du cyclone atlantique le plus intense $(882 \mathrm{hPa}$ pour Wilma, après Gilbert en 1988 qui avait atteint $888 \mathrm{hPa}$ ); du premier cyclone ayant touché l'Espagne (Vince, du 8 au 11 octobre)... La liste est longue ! Il faut malheureusement y ajouter le record du cyclone le plus meurtrier aux États-Unis depuis 1928, Katrina, qui a fait 1500 morts et le plus coûteux de l'histoire avec 80 milliards de dollars de dégâts.

Après cette saison 2005, et dans une décennie 1995-2005 beaucoup plus active que les deux précédentes, la question de savoir quelle était la responsabilité du réchauffement anthropique dans ces tendances est devenue essentielle pour la population et les scientifiques américains. Sous la pression médiatique, les études scientifiques relatives aux tendances observées ont fait l'objet d'un débat public peu compatible avec le recul scientifique nécessaire. Ainsi, deux écoles ont émergé du débat, celle qui considère qu'aucune tendance fiable ne peut être déduite de la base de données pour le bassin Atlantique, du fait de sa trop grande hétérogénéité, et celle qui essaie d'en trouver. En schématisant un peu : avant 2005 , les scientifiques de chacune de ces deux écoles débattaient

vivement pour savoir si le réchauffement climatique a déjà eu un impact sur l'activité cyclonique ou pas. Depuis 2005, la querelle s'est transformée en guerre de tranchées. Chaque article publié dans la presse scientifique est

repris et commenté par ses détracteurs dans les médias. Ainsi Webster et al. (2005) ont-ils, malgré eux, lancé la polémique en affirmant que le nombre total de cyclones de catégorie 4 et 5 a plus que doublé au cours de la période 1970-2004 (figure 5). Au cours de la même année, Emanuel (2005b) a montré que le potentiel destructif des cyclones, calculé à partir du cumul des vents portés au cube le long des trajectoires des cyclones, a dramatiquement augmenté depuis les années 1990, en parfaite association avec les TSM dans l'Atlantique tropical est (figure 6). En 2007, Webster et Holland (2007) récidivent et publient un article, dans lequel ils affirment que les discontinuités observées dans la base de données Hurdat

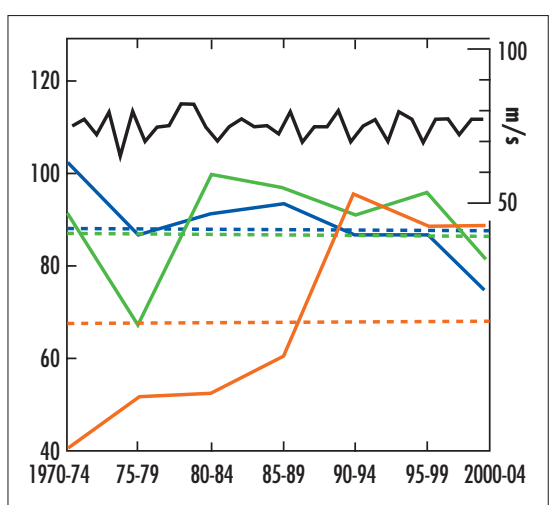

Figure 5 - Évolution du maximum global de vent (noir, en $\mathrm{m} / \mathrm{s}$ ) ; du nombre total de cyclones (bleu); de cyclones de catégorie 2 et 3 (vert) et de cyclones de catégorie 4 et 5 (rouge), par tranche de cinq ans, sur la période 1970-2004. Les traits pointillés représentent les moyennes par catégorie, du nombre de cyclones sur l'ensemble de la période1970-2004 (Webster et al., 2005).

(Hurricane Database) ne correspondent pas aux améliorations successives des moyens de mesure, mais qu'elles suivent plutôt l'évolution des TSM qui sont d'une autre base de données qui ne saurait présenter les mêmes discontinuités que Hurdat. Là encore, l'article est présenté et commenté dans la presse et s'en suit une discussion enflammée sur le forum dédié aux cyclones tropicaux. Ces trois articles de référence font, depuis 2005, couler beaucoup d'encre et nombreux sont les scientifiques à en remettre en cause le fondement. Les critiques se fondent sur la qualité de la base de données Hurdat et la variabilité naturelle à l'échelle décennale de l'activité cyclonique.

Figure 6 - Kerry Emanuel introduit une nouvelle manière de mesurer la force d'un ouragan : I'Indice de dissipation d'énergie (en anglais : Power Dissipation Index, PDI) qui prend en compte la durée et l'intensité d'un ouragan. Cet indice donne une information plus précise sur la réelle force destructrice des cyclones que sur leur fréquence ou les pertes économiques causées. Cette figure montre l'évolution annuelle du PDI (trait plein) et de la TSM de septembre (moyennée sur le domaine $6^{\circ}-18^{\circ} \mathrm{N}, 20^{\circ}-60^{\circ} \mathrm{W}$ ), sur le bassin Atlantique (traits pointillés). Les valeurs annuelles ont été préalablement lissées pour plus de lisibilité (Emanuel, 2005b).

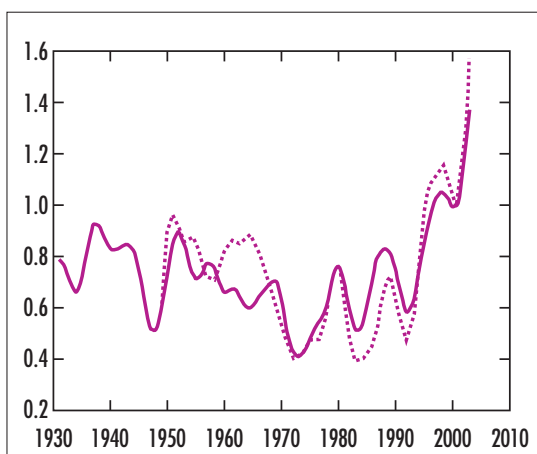


Hurdat, comme toutes les bases de données cycloniques, contient des informations dont la qualité s'est améliorée avec le temps, rendant délicat l'interprétation de résultats portant sur des périodes antérieures aux années 1970 (ère satellitaire). Si l'on restreint les études aux seules trente-cinq dernières années, il devient très difficile de déterminer si la tendance à la hausse observée est due à une réelle tendance imposée par le réchauffement anthropique ou à une manifestation de la variabilité décennale. Hurdat fait actuellement l'objet d'une réanalyse (Landsea et al., 2004) visant à l'homogénéiser sur des périodes plus longues, mais la qualité de la base restera toujours douteuse. En effet, comment estimer le nombre de cyclones qu'on a ratés dans le passé, ou les vents maxima atteints dans les phénomènes qu'on a observés ? Il faudra donc attendre encore quelques années avant de trancher définitivement le débat sur une éventuelle tendance, due à l'homme, de l'activité cyclonique. Il y a fort à parier que les dix prochaines années verront des éléments de réponse à cette question. En effet, la variabilité décennale observée jusqu'à présent (Roux, 2004) nous empêche de conclure sur la récente hausse de l'activité cyclonique. Mais si celle-ci est pilotée par ce type de variabilité, on devrait voir s'amorcer une tendance négative au cours de la prochaine décennie. Patience...

\section{Scénarios pour le XXle siècle}

En attendant le verdict des observations, il n'est pas interdit de spéculer sur l'avenir en étudiant ce que les modèles climatiques proposent comme tendances pour le XXI ${ }^{\mathrm{e}}$ siècle. Personne ne s'est d'ailleurs gêné pour le faire. Mais pour l'instant, on peut dire que les résultats ont du mal à converger, entre les scénarios climatiques qui indiquent un accroissement et ceux qui indiquent une baisse de l'activité. Ces divergences ont d'ailleurs été mentionnées dans le $4^{\mathrm{e}}$ rapport du Giec (IPCC, 2007), en précisant tout de même que certaines études à résolution élevée auraient tendance à s'accorder sur un affaiblissement du nombre de cyclones, mais avec une augmentation des cyclones intenses. Cette constatation n'est, pour l'instant, pas assez étayée pour en faire une généralité, mais elle n'a pas été démentie par les études les plus récentes. De même, il semblerait qu'indépendamment du signe de la tendance du nombre de cyclones dans les scénarios futurs, les précipitations associées aux phénomènes cycloniques verront leur intensité augmenter. Ce dernier point est important lorsque l'on sait que la majeure partie des victimes des cyclones périt à la suite des inondations engendrées par ces systèmes. Ces inondations sont principalement dues aux marées de tempêtes, mais dans certains endroits, les pluies cycloniques peuvent entraîner des glissements de terrain meurtriers. D'autres régions sont sensibles à l'apport en eau dû aux pluies cycloniques. Sans ces phénomènes, elles peuvent subir de graves sécheresses. Ce fut le cas de la Chine du Sud en 2004, $d^{\prime}$ 'après les conclusions du $6^{\text {th }} \mathrm{WMO}$ International Workshop on Tropical Cyclones, à San Jose (Costa Rica), en novembre 2006. D'autres régions peuvent subir le même sort, comme la Floride qui a besoin de l'apport en eau des cyclones pour équilibrer son bilan d'eau.

En ce qui concerne le CNRM, les études, menées sur le sujet depuis dix ans, ont concerné aussi bien l'approche par les conditions de grande échelle que l'approche directe. Cette dernière a été menée ces dernières années, dans le cadre du projet français Imfrex (Impact des changements anthropiques sur la fréquence des phénomènes extrêmes de vent, de température et de précipitations ; cf. la page web du projet : http://medias.cnrs.fr/ imfrex/web/index.fr.php). À l'aide de simulations à forte résolution en mode forcé, avec des anomalies de TSM issues de scénarios climatiques à basse résolution, nous avons développé une méthodologie de suivi de trajectoires qui s'est montrée intéressante (Chauvin et al., 2006). Différents types de simulations ont été utilisés, ainsi que différentes anomalies de TSM.

La première simulation est issue du scénario climatique du CNRM, utilisé antérieurement au précédent exercice Giec et l'autre provient d'un scénario du Hadley Centre (Grande-Bretagne). Le coût d'une simulation climatique globale à une résolution de $50 \mathrm{~km}$ est suffisamment élevé pour que ce genre d'exercice ne puisse pas encore se répéter souvent. Pour cette étude, deux simulations de dix ans chacune (une pour le climat présent et une pour la fin du XXI ${ }^{\mathrm{e}}$ siècle) ont été réalisées avec une telle résolution. Étant donné que notre domaine d'étude se restreignait à l'océan Atlantique, nous avons également eu recours à des expériences complémentaires en grille basculée/étirée. Cette méthodologie consiste (comme nous l'avons déjà mentionné plus haut), pour une résolution globale donnée, à étirer la grille du modèle de manière à resserrer les mailles sur la région concernée, au détriment de l'antipode qui voit sa résolution dégradée. Pour ce faire, le pôle de la grille est basculé pour venir se placer au centre du domaine d'étude. À l'aide de cette configuration du modèle Arpège, nous avons réalisé plusieurs simulations de trente ans chacune (une pour le présent et une pour chacune des anomalies futures de TSM produites par les scénarios couplés à plus basse résolution). Comme la technologie du «basculé/ étiré »s'est révélée particulièrement adaptée à ce genre de problématique, l'économie sur le temps de calcul a permis d'allonger significativement les simulations. Cela nous a permis également de comparer les résultats obtenus pour chacune des deux grilles (uniforme et basculée/étirée) et de constater, dans les deux cas, que la réponse de l'activité cyclonique à un scénario d'augmentation du $\mathrm{CO}_{2}$ dépendait plus de la distribution géographique de l'anomalie de TSM (c'est-à-dire la manière dont celle-ci se répartit dans les différents bassins océaniques) que de son intensité. En effet, des anomalies sur les gradients de TSM pilotent des réponses atmosphériques plus marquées qu'une augmentation uniforme de TSM. L'anomalie de TSM du scénario du CNRM est plus faible que celle du Hadley Centre (principalement à cause du scénario différent d'accroissement des gaz, sélectionné dans chacune des expériences). En revanche, elle est uniformément répartie sur le bassin Atlantique, ce qui n'est pas le cas de l'anomalie produite par le Hadley Centre. Le nombre de trajectoires pour l'anomalie CNRM augmente dans le futur alors que celui des trajectoires obtenues pour l'anomalie du Hadley Centre diminue. Les deux expériences s'accordent sur une augmentation de cyclogenèse dans le golfe du Mexique. On interprète celle-ci avec précaution, étant donnée la surestimation du nombre de trajectoires que le modèle produit dans le climat présent, sur cette zone. Néanmoins, la faible profondeur de cette mer pourrait expliquer des variations de TSM plus marquées du fait d'une inertie thermique moindre, pouvant favoriser plus de déclenchements. La simulation utilisant les anomalies de TSM du Hadley Centre présente une structure d'anomalie de TSM dipolaire est/ouest sur l'Atlantique ainsi qu'une anomalie positive marquée sur le Pacifique est, qui ont pour effet de générer une augmentation du cisaillement vertical du vent horizontal, qui est à l'origine de la baisse d'activité sur l'Atlantique ouest. La figure 7 représente l'anomalie de densité de jours de cyclones pour chacune des deux expériences. L'absence de différence sur la partie est de l'océan Atlantique n'est probablement qu'une faiblesse de la méthode de détection des trajectoires, laquelle a tendance à minimiser le nombre de jours de cyclones sur cette région de cyclogenèse. En utilisant l'approche 
indirecte et l'indice convectif de cyclogenèse mentionné plus haut (Royer et al., 1998), les deux expériences s'accordent sur une légère augmentation dans la zone comprise entre $50^{\circ} \mathrm{W}$ et $20^{\circ} \mathrm{W}$ aux alentours de $10^{\circ} \mathrm{N}$.

Au-delà des antagonismes soulignés plus haut, les deux expériences s'accordent sur l'anomalie des précipitations associée au phénomène cyclonique qui augmenterait dans le climat futur. Nous avons attribué cette augmentation à l'accroissement de l'évaporation dans un climat plus chaud. En revanche, seule une légère augmentation de vents, non significative, est perceptible dans ces simulations, contrairement à d'autres études qui indiquent une augmentation plus marquée.

La science avance très vite dans ce domaine. Récemment, une équipe japonaise a réalisé deux simulations globales de dix ans chacune à $20 \mathrm{~km}$ de résolution (Oouchi et al., 2006) sur le Earth Simulator japonais, dans lesquelles les TSM sont prescrites. La simulation du climat présent est forcée par la climatologie observée de TSM alors que, pour celle du climat futur, on y ajoute une anomalie calculée par une simulation couplée océan/atmosphère à basse résolution. Leurs résultats indiquent une baisse de l'activité cyclonique avec augmentation des cyclones intenses. Ces résultats sont confirmés par une étude récente de Bengtsson et al. (2007). Bien entendu, ces expériences dépendent fortement de l'anomalie calculée par le modèle à basse résolution. De plus, de telles résolutions seront difficiles à généraliser dans un proche avenir, du fait de leur coût en temps de calcul.

En ce qui concerne l'approche indirecte, outre l'article de 1998 (Royer et al., 1998), nous avons entrepris récemment, au sein de l'équipe, une étude systématique des scénarios de la base de données du Giec, en calculant le CYGP pour chaque période et chaque modèle. Les premiers résultats montrent encore une large dispersion dans les réponses des modèles engagés dans le dernier exercice (Royer et Chauvin, 2008). Ces résultats s'inscrivent dans les conclusions du $4^{\text {e }}$ rapport du Giec qui fait état de cette dispersion. Ils semblent confirmer également un des résultats obtenus avec Arpège-Climat en 2006 (Chauvin et al, 2006), à savoir qu'il pourrait y avoir un lien entre la réponse de l'activité cyclonique et la structure géographique de l'anomalie de TSM dans les modèles. Cette approche indirecte a également fait l'objet d'un récent article (Caron et Jones, 2007) calculant le CYGP pour un sous-

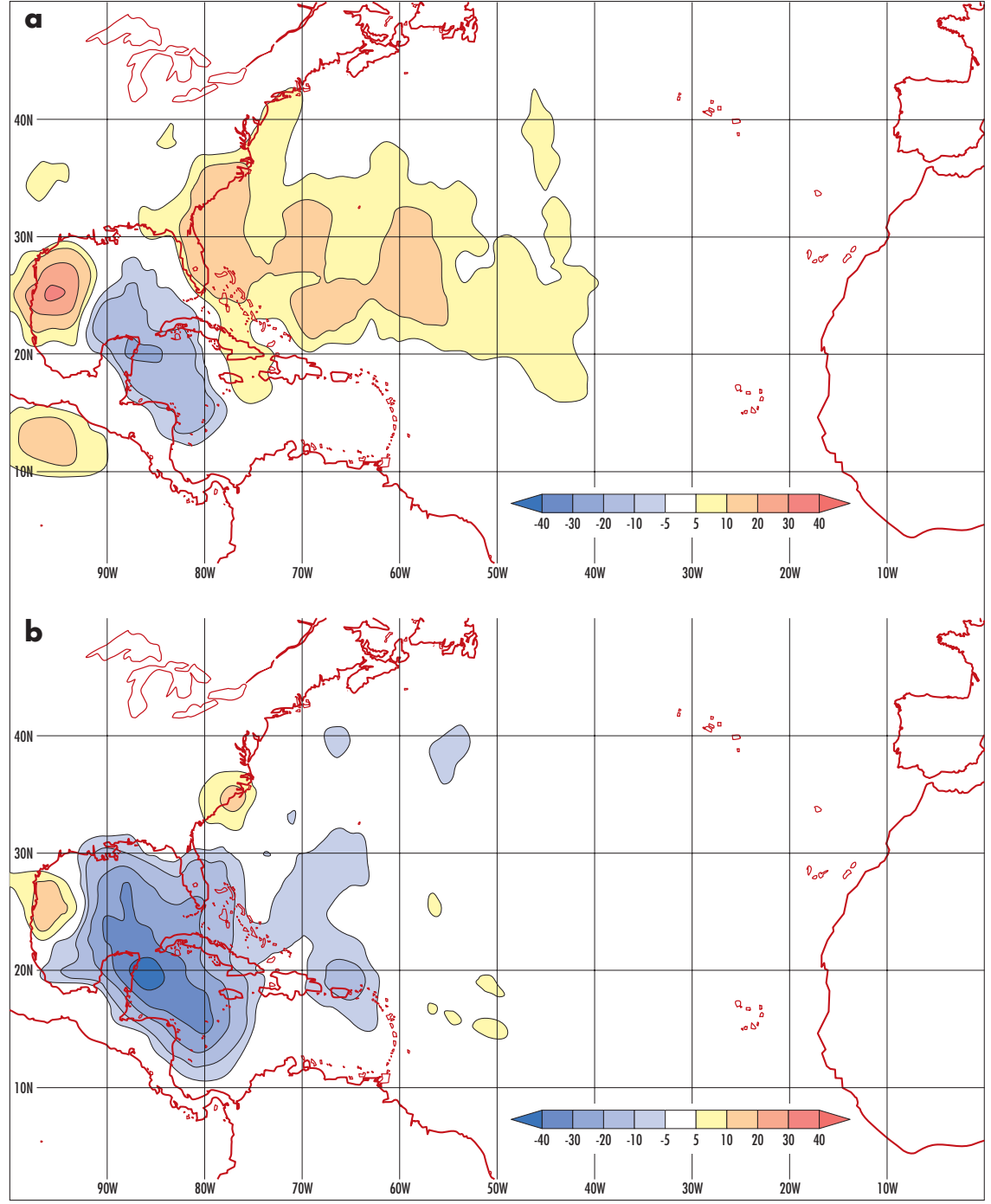

Figure 7 - Anomalie du nombre de jours de cyclones entre le climat futur (2070-2099) et présent (1970-1999) pour les simulations effectuées au CNRM avec des conditions de surface provenant des scénarios du CNRM (a) et du Hadley Centre (b). Les densités sont exprimées en nombre de cyclones par 5 degrés par vingt ans.

ensemble des simulations produites pour le Giec. Moyennant une sélection des modèles dont le climat présent est le plus proche des observations, les auteurs trouvent une légère augmentation de l'indice CYGP moyen, et ce pour les différents scénarios du Giec. Géographiquement, l'anomalie est principalement localisée dans le Pacifique nord. Pris séparément, ces modèles présentent, néanmoins, des divergences marquées.

D'autres indices ont été élaborés depuis quelques années pour rendre compte de l'activité cyclonique. On peut mentionner le plus récent, qui concurrence le CYGP : le Genesis Potential Index (GPI), élaboré par Kerry Emanuel et ses collègues, et dont l'utilisation dans les scénarios est prometteuse (Camargo et al., 2007c). Cet indice repose sur à peu près les mêmes ingrédients que le CYGP, les précipitations convectives étant remplacées par l'intensité potentielle, une mesure de l'énergie disponible pour la cyclogenèse et l'humidité des couches moyennes de la troposphère. La confrontation de ces différents indices ne peut que conduire à une meilleure compréhension du phénomène.

Un autre type d'approche (Camargo et al., 2007 a et b) semble prometteur pour étudier l'impact du réchauffement anthropique sur l'activité cyclonique. Il s'agit de distinguer, par des techniques statistiques de type « cluster », différents types dans l'ensemble des trajectoires cycloniques, qu'elles soient extraites des bases de données observées ou de simulations à haute résolution. Pour le Pacifique nord-ouest, Camargo et al. (2007a) ont déterminés sept clusters, dont on peut calculer ensuite les différentes caractéristiques : location de genèse, intensité, trajectoire, période d'apparition, etc. En étudiant les relations entre ces clusters et les conditions de grande échelle, on peut déterminer leur sensibilité au climat (Camargo, 2007b). En utilisant cette méthodologie, plutôt que de 
chercher comment le nombre de cyclones va évoluer dans le futur, on pourrait estimer dans quelle mesure la répartition des trajectoires pourrait changer dans un climat plus chaud (sous l'hypothèse que la décomposition en clusters reste stable dans le climat futur, ce qui n'est pas acquis). De plus, la méthode semble plus robuste qu'un simple comptage des cyclones dans les simulations.

\section{Conclusion}

Il ne fait aucun doute que la vulnérabilité des sociétés au risque cyclonique rend l'étude de son évolution essentielle pour les générations futures. La vigilance des populations pouvant se relâcher à l'occasion d'une longue période d'accalmie, comme celle qu'à connu l'Atlantique au cours des années 1970-1990, le retour à une activité normale ou forte peut avoir des conséquences catastrophiques. La saison 2005 et le cyclone Katrina, en particulier, ont marqué les esprits, tout d'abord par la violence des phénomènes qui se sont produits, mais également par le manque de préparation des populations et des institutions publiques à affronter de telles catastrophes. Katrina n'était pourtant plus qu'un cyclone de catégorie 3 (110 nœuds tout de même !) lorsqu'il a atteint les côtes de la Louisiane, le 29 août 2005 à 11 h 10 UTC, après avoir atteint son intensité maximale de 150 nœuds la veille à 18 h 00 UTC (catégorie 5) [figures 8 et 9]. Le rapport du National Hurricane Center du 20 décembre 2005, mis à jour le 10 août 2006, fait état de 1833 victimes dont 1500 directement causées par le passage du cyclone ; la ville de la Nouvelle-Orléans, où l'on déplore la majorité des victimes, comptait 500000 habitants, alors que l'agglomération qui l'entoure en comptait 1,4 million. Cet événement a malheureusement rappelé qu'une épée de Damoclès est dressée audessus de la tête de nombreux habitants des côtes soumises au risque cyclonique, et ce partout dans le monde. Il faut néanmoins mentionner que, même dans des pays où la culture du risque n'est pas forcément développée, les mesures préventives

Figure 8 - Trajectoire de l'ouragan Katrina qui a frappé la Louisiane en août 2005.

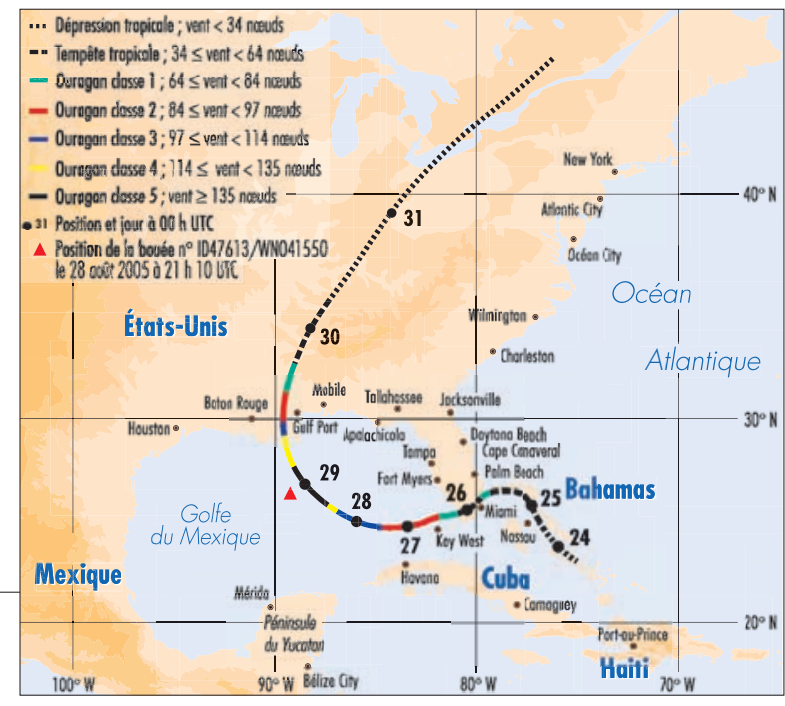

Figure 9 - Carte des vents maximaux en nœuds estimés entre le 28 août à 18 h UTC où l'ouragan Katrina de classe 3 passe sur le golfe du Mexique et le 31 août 2005 à 00 h UTC où Katrina, déclassée en dépression tropicale est située dans les terres entre vers $40^{\circ} \mathrm{N}$ et $85^{\circ} \mathrm{W}$.

(construction de refuges, implantation d'arbres en bordure de mer pour minimiser les effets de l'onde de tempête, alertes précoces...) et d'éducation aux risques commencent à donner quelques résultats.

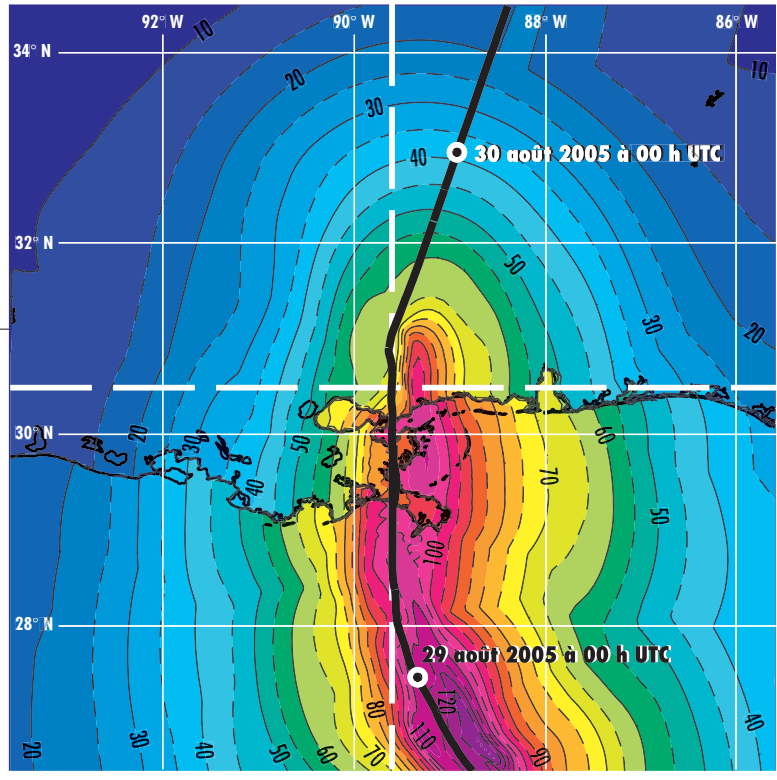
L'exemple du cyclone Sidr de novembre 2007, qui a fait environ 3500 morts au Bangladesh, est à mettre en regard des précédentes catastrophes de 1970 (Bhola, novembre 1970) et 1991 (Gorky, avril 1991) dans ce même pays, qui occasionnèrent chacun des centaines de milliers de morts. S'il reste beaucoup à faire, il semblerait néanmoins que l'on soit sur la bonne voie.

Les scientifiques s'affrontent actuellement pour savoir si le nombre ou l'intensité des cyclones a déjà augmenté, ou va augmenter, avec l'accroissement des gaz à effet de serre dans l'atmosphère. Les débats sont houleux, parfois à la limite de la correction. Il ne faut pourtant pas oublier que, comme le dit Boris Vian dans La Java des bombes atomiques : «Voilà des mois et des années Que j'essaye d'augmenter La portée de ma bombe Et je n'me suis pas rendu compt' Que la seul'chos' qui compt' C'est l'endroit où c'qu'ell' tombe. »"

Les cyclones sont des bombes naturelles dont le potentiel destructeur dépend fortement de l'endroit où ils atterrissent. De ce fait, même dans un contexte où 1 'intensité moyenne diminuerait légèrement, le potentiel destructeur pourrait très bien augmenter pour de multiples raisons, dont l'évolution climatique ne serait qu'une infime partie.

Du côté des scientifiques, indépendamment de la polémique sur une tendance éventuelle de l'activité cyclonique observée sur le $\mathrm{XX}^{\mathrm{e}}$ siècle, il semble qu'à l'avenir, il faudra résoudre l'incertitude sur la tendance prévue par les modèles pour le $\mathrm{XXI}^{\mathrm{e}}$ siècle. Pour l'instant, les études ne convergent pas, mais une possibilité, qui se dessine, est une baisse du nombre de cyclones avec, en contrepartie, une augmentation des cyclones intenses. En d'autres termes, l'évolution des conditions de grande échelle pourrait devenir moins favorable au déclenchement cyclonique, mais la hausse des TSM permettrait une alimentation accrue des systèmes existants.

Au-delà de la question du nombre ou de l'intensité, des études commencent à s'intéresser à la manière dont les trajectoires pourraient être infléchies par le réchauffement climatique. Cette approche du problème est vraiment primordiale car, contrairement à la question du nombre ou de l'intensité, le changement de trajectoire pourrait avoir comme effet de toucher des populations jusquelà épargnées par les phénomènes cycloniques. Rappelons que l'océan Atlantique sud a connu son premier ouragan officiel en mars 2004 et que l'Espagne en a vu un de près en octobre 2005 (Vince). De même, la possibilité de développements plus fréquents de 
phénomènes subtropicaux intenses, tels que ceux observés en fin de saison cyclonique sur différents bassins, est une éventualité à considérer sérieusement. Les risques les plus importants dans les années à venir pourraient bien se trouver dans ces zones plutôt que celles qui connaissent déjà aujourd'hui les affres du « vent divin ».

\section{Remerciements}

Cet article s'est beaucoup appuyé sur le magnifique livre de Kerry Emanuel, Divine Wind (2005a). On y découvre une information très complète sur les cyclones, le tout illustré de figures et de textes relatifs à ces phénomènes. Une lecture à recommander.
À noter aussi la contribution de l'article de F. Roux et N . Viltard (1997), apportant une information précieuse et en français. Il faut également rendre hommage à l'ensemble de nos collègues des équipes Climat du CNRM qui contribuent activement aux exercices du Giec et aux progrès scientifiques dans ce domaine.

\section{Bibliographie}

\footnotetext{
- Ayrault F. et A. Joly, 2000 : Une nouvelle typologie des dépressions météorologiques : classification des phases de maturation. C. R. Acad. Sci., Paris, série lla 330, 167-172.

Bengtsson L., K. I. Hodges, M. Esch, N. Keenlyside, L. Kornblueh, J.-J. Luo et T. Yamagata, 2007 : How may tropical cyclones change in a warmer climate? Tellus, 59A, 539-561.

- Camargo S. J., A. W. Robertson, S. J. Gaffney, P. Smyth et M. Ghil, 2007a : Cluster Analysis of Typhoon Tracks. Part I: General Properties. J. Climate, $20,3635-3653$.

- Camargo S. J., A. W. Robertson, S. J. Gaffney, P. Smyth et M. Ghil, 2007b : Cluster Analysis of Typhoon Tracks. Part II: Large scale circulation and ENSO. J. Climate, 20, 3654-3676

Caron L.-P. et G. J. Jones, 2007 : Analysing past, present and future tropical cyclone activity as inferred from an ensemble of coupled global climate models. Tellus, 60A, 80-96.

- Chauvin F., J.-F. Royer et M. Déqué, 2006 : Response of hurricane-type vortices to global warming as simulated by Arpege-Climat at high resolution. Climate Dyn., 27(4), 377-399 D0I:10.1007/s00382-006-0135-7

- Davis L., 2002 : Natural Disasters-Revised edition. Facts on file, 420 p.

- Dvorak V. F., 1975 : Tropical cyclone intensity analysis and forecasting from satellite imagery. Mon. Wea. Rev., 103, 420-430.

- Dvorak V. F., 1984 : Tropical cyclone intensity analysis using satellite data. NOAA Tech. Report NESDIS 11. Available from NOAA/NESDIS, 5200 Auth Rd., Washington DC, 20233, 47 p.

Emanuel K. A., 1986 : An air-sea interaction theory for tropical cyclones. Part I: Steady-state maintenance. J. Atmos. Sci., 43, 585-604.

- Emanuel K. A., 1988 : The maximum intensity of hurricanes.J. Atmos. Sci., 45, 1143-1155.

- Emanuel K. A., 2005a : Divine Wind. Oxford University Press, 285 p.

Emanuel K. A., 2005b : Increasing destructiveness of tropical cyclones over the past 30 years. Nature, 436, 686-688.

- Fitzpatrick P. J., 2006 : Hurricanes. A reference handbook - Second edition. Contemporary World Issues, $412 \mathrm{p}$

- Gray W. M., 1968 : Global view of the origin of tropical disturbances and storms. Mon. Wea. Rev., 96, 669-700.

- Holland G. J. et P. J. Webster, 2007 : Heightened tropical cyclone activity in the North Atlantic: natural variability of climate trend?. Phil. Trans. R. Soc. A. En cours de publication.

IPCC, 2007 : Climate Change 2007. IPCC Fourth Assessment Report. Cambridge University Press.

Knabb R. D., J. R. Rhome et D. B. Brown, 2005 : Tropical cyclone report. Hurricane Katrina, 23-30 August 2005. Ce rapport est disponible à l'adresse : http://www.nhc.noaa.gov/2005atlan.shtml.

Landsea C. W., C. Anderson, N. Charles, G. Clark, J. Dunion, J. Partagas, P. Hungerford, C. Neumann, et M. Zimmer, 2004 : The Atlantic hurricane database re-analysis project: Documentation for the 1851-1910 alterations and additions to the Hurdat database. Hurricanes and Typhoons: Past, Present, and Future de R.J. Murnane et K.-B. Liu. Editors, Columbia University Press, New York, 177-221.

Landsea C. W., 2007 : Counting Atlantic tropical cyclones back to 1900. EOS, 88, 18, 197-202.

- Liu K.-B., 2004 : Paleotempestology: Principles, methods, and examples from Gulf Coast Lake Sediments. Hurricanes and Typhoons: Past, Present, and Future, de R.J Murnane et K.-B. Liu Editors, Columbia University Press, New York, 13-57.

- Oouchi K., J. Yoshimura, H. Yoshimura, R. Mizuta, S. Kusunoki et A. Noda, 2006 : Tropical Cyclone Climatology in a Global-Warming Climate as Simulated in a 20km-Mesh Global Atmospheric Model: Frequency and Wind Intensity Analyses. J. Met. Soc. Japan, 84, 259-276.

Renaut D., 2005 : Katrina a semé la désolation aux États-Unis. La Météorologie, 8 série, 51, 2-3.

- Roux F. et N. Viltard, 1997 : Les cyclones tropicaux. La Météorologie, 8 e série, 18, 9-33.

Roux F., 2005 : Les cyclones atlantiques en 2004 : chronique d'une année annoncée. La Météorologie, 8e série, 48, 23-27.

- Royer J.-F., Chauvin F., Timbal B., Araspin P. et Grimal D., 1998 : A GCM study of the impact of greenhouse gas increase on the frequency of occurrence of tropical - cyclones. Clim. Change, 38, 307-343.

- Royer J.-F. et F. Chauvin, 2008 : Response to tropical cyclogenesis to global warming in an IPCC AR-4 scenario assessed by a modified yearly genesis parameter. - À paraître dans Hurricanes and Climate Change, J. Elsner (ed.), Springer.

- Sugi M., A. Noda et N. Sato, 2002 : Influence of the global warming on Tropical cyclone climatology: An experiment with the JMA global model. J. Me. SoC. Japan, $80,249-272$.

- Webster P. J., G. J. Holland, J. A. Curry et H.-R. Chang, 2005 : Changes in Tropical Cyclone Number, Duration, and Intensity in a Warming Environment. Science, $309,1844-1846$.

Willoughby H. E., D. P. Jorgensen, R. A. Black et S. L. Rosenthal, 1985 : Project Stormfury: a scientific chronicle 1962-1983. Bull. Amer. Meteor. Soc., 66, 505-514.

Webster P.J., G.J. Holland, J.A. Curry et H.-R. Chang, 2005 : Changes in Tropical Cyclone Number, Duration, and Intensity in a Warming Environment. Science, 309, 1844-1846.

Xu K. et K. A. Emanuel, 1989 : Is the tropical atmosphere conditionally unstable? Mon. Wea. Rev., 117, 1471-1479.
} 